MITSUBISHI ELECTRIC RESEARCH LABORATORIES

http://www.merl.com

\title{
Comparative Analysis of Pilot-Assisted Distributed Co-Phasing Approaches in Wireless Sensor Networks
}

\author{
Chaythanya, K.V.; Annavajjala, R.; Murthy, C.R. \\ TR2011-059 August 2011
}

\begin{abstract}
This paper compares and analyzes the performance of distributed cophasing techniques for uplink transmission over wireless sensor networks. We focus on a time-division duplexing approach, and exploit the channel reciprocity to reduce the channel feedback requirement. We consider periodic broadcast of known pilot symbols by the fusion center (FC), and maximum likelihood estimation of the channel by the sensor nodes for the subsequent uplink co-phasing transmission. We assume carrier and phase synchronization across the participating nodes for analytical tractability. We study binary signaling over frequency flat fading channels, and quantify the system performance such as the expected gains in the received signal-to-noise ratio (SNR) and the average probability of error at the FC, as a function of the number of sensor nodes and the pilot overhead. Our results show that a modest amount of accumulated pilot SNR is sufficient to realize a large fraction of the maximum possible beamforming gain. We also investigate the performance gains obtained by censoring transmission at the sensors based on the estimated channel state, and the benefits obtained by using maximum ratio transmission (MRT) and truncated channel inversion (TCI) at the sensors in addition to cophasing transmission. Simulation results corroborate the theoretical expressions and show the relative performance benefits offered by the various schemes.
\end{abstract}

IEEE Transactions on Signal Processing

This work may not be copied or reproduced in whole or in part for any commercial purpose. Permission to copy in whole or in part without payment of fee is granted for nonprofit educational and research purposes provided that all such whole or partial copies include the following: a notice that such copying is by permission of Mitsubishi Electric Research Laboratories, Inc.; an acknowledgment of the authors and individual contributions to the work; and all applicable portions of the copyright notice. Copying, reproduction, or republishing for any other purpose shall require a license with payment of fee to Mitsubishi Electric Research Laboratories, Inc. All rights reserved.

Copyright (c) Mitsubishi Electric Research Laboratories, Inc., 2011

201 Broadway, Cambridge, Massachusetts 02139 



\title{
Comparative Analysis of Pilot-Assisted Distributed Co-Phasing Approaches in Wireless Sensor Networks
}

\author{
Krishna Chaythanya K. V. ${ }^{1}$, Ramesh Annavajjala ${ }^{2}$ and Chandra R. Murthy ${ }^{1}$ \\ ${ }^{1}$ Dept. of ECE, Indian Institute of Science, Bangalore, India \\ ${ }^{2}$ Mitsubishi Electric Research Laboratories, Cambridge, MA, 02139, USA \\ Email: krishnac@ece.iisc.ernet.in, ramesh.annavajjala@gmail.com, cmurthy@ece.iisc.ernet.in
}

\begin{abstract}
This paper compares and analyzes the performance of distributed cophasing techniques for uplink transmission over wireless sensor networks. We focus on a time-division duplexing approach, and exploit the channel reciprocity to reduce the channel feedback requirement. We consider periodic broadcast of known pilot symbols by the fusion center (FC), and maximum likelihood estimation of the channel by the sensor nodes for the subsequent uplink co-phasing transmission. We assume carrier and phase synchronization across the participating nodes for analytical tractability. We study binary signaling over frequencyflat fading channels, and quantify the system performance such as the expected gains in the received signal-to-noise ratio (SNR) and the average probability of error at the $\mathrm{FC}$, as a function of the number of sensor nodes and the pilot overhead. Our results show that a modest amount of accumulated pilot SNR is sufficient to realize a large fraction of the maximum possible beamforming gain. We also investigate the performance gains obtained by censoring transmission at the sensors based on the estimated channel state, and the benefits obtained by using maximum ratio transmission (MRT) and truncated channel inversion (TCI) at the sensors in addition to co-phasing transmission. Simulation results corroborate the theoretical expressions and show the relative performance benefits offered by the various schemes.
\end{abstract}

Index Terms-Channel reciprocity, distributed co-phasing, phase estimation errors, limited feedback, sensor censoring, channel inversion.

\section{INTRODUCTION}

Recently, there has been a growing interest in the research community in the area of wireless sensor networks (WSN). A WSN is loosely defined as a collection of wireless nodes dedicated to perform a specialized task [1], [2]. Some of the applications of WSNs include environment and habitat monitoring, health care aspects such as patient monitoring and disability assistance, home automation and security, quality control and inventory management.

The idea of distributed transmit beamforming (DTB) as a mechanism for cooperative communication from a set of sensors to a fusion center (FC) has been explored by various researchers in the context of information transmission over a correlated sensing field and over wireless ad hoc networks [3] - [16]. With DTB, a cluster of densely deployed sensor nodes can act as a virtual transmit antenna array to transmit a correlated observation to the FC, thereby providing coherent

A portion of this work was presented at the 6th IEEE Sensor, Mesh and Ad Hoc Communications and Networks Conference (SECON 2009), Italy. combining gain and improving the energy efficiency of the sensor nodes. An initial investigation of the potential gains of DTB on non-fading channels is conducted in [3], and the feasibility of DTB on wireless fading channels is investigated in [4]. In the context of wireless ad hoc and sensor networks, the distribution of the virtual antenna array beam pattern is studied in [5] for uniformly distributed nodes and in [6] for Gaussian distributed nodes. [7] and [8] propose methods for information-sharing among wireless and ad-hoc networks which then use DTB. The optimal transmission weights at the sensors to maximize received SNR are found in [8]. In [9], optimal power allocation for relays using DTB is considered in a single-source-multiple-relay setup when perfect CSI is available at all the transmitters and receivers. The optimal transmit weights when only second order statistics of the channel state is available are found in [10]. [11] presents a performance analysis of DTB with quantized feedback.

Some of the practical challenges that need to be overcome in realizing the full potential of DTB are carrier-phase and symbol synchronization across the sensor nodes, estimation of fading channel parameters for coherent detection at the FC, and energy efficient operation when the nodes are subjected to a short-term power constraint. In the context of nonfading channels, [3] proposes a master-slave approach to time synchronization. With a per-node long-term average transmit power constraint, [4] extends the master-slave approach in [3] to wireless fading channels. Carrier phase synchronization with two autonomous nodes is studied in [12] and [13], and the impact of channel errors on the transmit beam pattern is reported in [7]. A random phase adjustment by each sensor node for feedback-based control is studied in [14], and [16] proposes deterministic perturbation and a 1-bit feedbackbased approach, and performs a simulation based study of the schemes. Distributed beamforming with low-rate feedback from the sensor nodes to the fusion node is considered in [17], where the authors analyze the convergence behavior of an adaptive distributed beamforming scheme. In [18], the authors analyze the convergence behavior of a class of singlebit feedback-based phase alignment algorithms and propose improvements to existing algorithms.

In this paper, we are interested in the comparative analysis of distributed co-phasing (DCP) systems. Our focus is on analyzing the relative performance of various schemes 
of transmission using the DCP approach and the effect of channel estimation errors on the performance. We focus on a time-division duplexing (TDD) model to exploit the channel reciprocity for acquiring channel knowledge at the transmitting nodes with minimal overhead. reduction. Note that channel reciprocity requires well-calibrated transmit and receive RF chain components. Also, all the sensors and the FC need to be carrier phase, frequency and timing synchronized. We estimate the channel at the transmitting nodes using a pilot signal from the receiver, which clearly requires a significantly smaller training and feedback overhead than, for example, a frequency duplexing division approach.

Carrier phase and frequency synchronization of participating terminals is a widely used assumption in the fields of cooperative communications, distributed space-time coding, physical layer fusion-based literature. The effects of errors in carrier phase and frequency synchronization and timing synchronization on channel estimation in a reciprocal system such as considered here are discussed in [4] and [19]. Masterslave architectures to achieve carrier-phase synchronization are proposed and analyzed in [3] and [4]. Schemes for synchronization of two-sources are proposed in [12], [13]. A masterslave ping-pong based synchronization scheme is proposed in [19]. It is argued in [4] that though it is challenging to achieve such synchronization, it is potentially feasible and that moderately large phase errors due to synchronization errors do not significantly affect the performance. Since our focus is on the performance comparison of transmission schemes, we assume that such synchronization can be achieved using one of the above mentioned schemes. To achieve synchronization, we consider a super-frame structure that contains a phase synchronization frame followed by multiple data frames. We assume that one of the existing algorithms mentioned above is used to achieve the necessary synchronization. Since there is one synchronization frame every super-frame, the participating terminals carry out synchronization once every super-frame. The data frames within a super-frame contain downlink pilot symbols (see Fig. 1), that are used to estimate the phase of the complex channel gain, followed by uplink data transmission from the sensors to the FC. The modeling and analysis of synchronization induced errors is beyond the scope of this paper and is relegated to future work.

The following are the main contributions of this paper. We analyze and compare the performance of four schemes for information transmission from the sensors to the FC and the effect of channel estimation errors on the performance. The schemes differ in the power allocation and the way they exploit the estimated channel gains. The first two schemes are constant power allocation schemes, where whenever the sensors transmit, they transmit at a fixed power. We term the first scheme as the baseline distributed co-phasing (DCP). Here, we study the distribution of the channel phase estimation error at the sensors and the probability of signal corruption at the FC due to imperfect channel estimation. For simplicity, we restrict the analysis to coherent binary modulation on independent Rayleigh fading channels, and derive closedform expressions for the average received SNR. We present a simple-to-evaluate expression for the average bit error rate

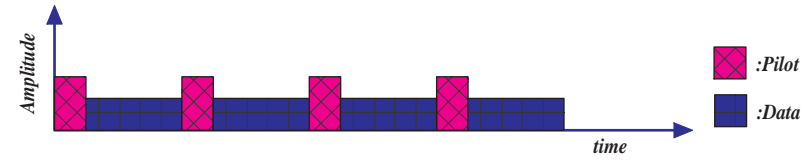

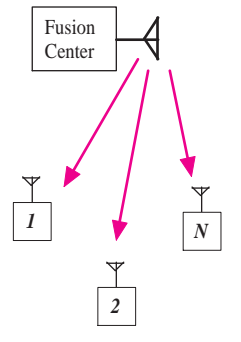

a) Phase-1: DL Sounding

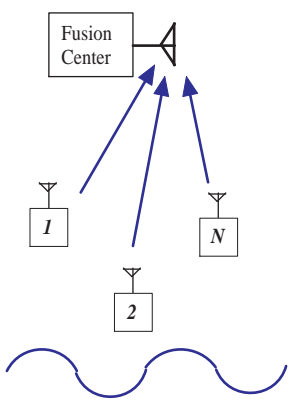

b) Phase-2: UL Transmission
Fig. 1. Per-node phase estimation via downlink broadcast pilot. Multiple access data transmission via uplink.

(BER) at the FC based on an improved Gaussian approximation (IGA) [20]. Our short-term power constraint based results show that the actual achievable beamforming gains are between 2 to $3 \mathrm{~dB}$ below that of the gains reported in [4] which employs power allocation across sensors. However, note that a power allocation as in [4] implies that one has to employ RF components that can support a wide dynamic range of transmit powers and that nodes need to have a mechanism for sharing the power allocation information between them. In contrast, the distributed co-phasing has the advantage that the nodes transmit at a fixed power, and can therefore be implemented with relatively inexpensive RF components.

Next, we analyze a scheme for DTB based on the censoring sensors approach. We constrain the sensors to transmit only when the channel gain is sufficiently high. Our approach to censoring sensors is different from that in [21], where the sensors are censored based on the likelihood ratios. In our model, the sensors whose channels to the FC are in deep fades do not contribute significantly to the decision made at the FC. Hence, these sensors do not transmit and save their power. We impose a long term but individual power constraint on the sensors. The sensors boost their power based on the probability that they transmit at any given instant. We derive closed form expressions for the average received SNR for Rayleigh fading channels. It is found, somewhat surprisingly, that the censoring sensors approach offers a very marginal performance improvement over the baseline DCP scheme.

The next two schemes allocate variable power per transmitted symbol depending on the estimated channel gain at the sensor. Here, we impose long-term power constraints at the each sensor. We focus on two popular schemes, namely, the maximum ratio transmission (MRT) and the truncated channel inversion (TCI) scheme. In MRT, sensors allocate their power proportional to the estimated channel power gain, while satisfying an average power constraint. In TCI, the 
sensors allocate a power inversely proportional to the estimated channel power gains, but do not transmit when the estimated channel gain is below a certain threshold. We derive analytical expressions for the average received SNR at the FC for Rayleigh fading channels with MRT and TCI. Monte-Carlo simulations validate our analysis in all cases.

The rest of this paper is organized as follows. In Section II, we introduce our system model. Performance analysis of the DCP system is presented in Section III, wherein we derive expressions for the distribution of the phase error at the sensor nodes, the probability of signal corruption, the average received SNR and the average probability of error. Expressions for average received SNR are derived when the sensors censor themselves based on the channel state in Section IV. In Section V, expressions for average received SNR for TCI and MRT are derived. Results and discussions are presented in Section VI, and conclusions are provided in Section VII.

\section{SySTEM MODEL}

We consider a sensing field deployed with $N$ sensor nodes. The observations across the sensor nodes, depending upon the nature of the sensing field, can be described by a joint probability distribution function. A binary modulation is employed to transmit the sensor observations to the FC. The sensor nodes and the FC are all assumed to have a single transmit and receive antenna. As discussed in Section I, we assume for analytical tractability, that the local oscillator (LO) phases of different nodes are synchronized and that the estimated channel gains are corrupted by Gaussian noise only. This can be achieved by adopting a super-frame structure containing a phase synchronization frame to periodically synchronize the LO phases of the sensors nodes against variations in temperature and the environment; and employing the previously proposed master-slave techniques in [3], [4], [12].

We consider the reciprocal TDD system model illustrated in Fig. 1. Each data frame contains $N_{P}$ pilot symbols sent by the FC to the sensors in the downlink, followed by a simultaneous transmission from the $N$ sensors, using DTB, over $N_{D}$ channel uses (i.e., $N_{D}$ data symbols are sent). Assuming a narrow-band transmission between the sensor nodes and the FC, the low-pass equivalent complex-valued channel between the $k$ th sensor and the FC is denoted by $g_{k}=\alpha_{k} e^{j \theta_{k}}$, where $\alpha_{k}$ is the fade amplitude and $\theta_{k}$ is the phase of $g_{k}$. The channel is assumed to be quasi-static over a frame duration of $N_{P}+N_{D}$ symbols, and varies independently across the data frames. The channel gains $g_{k}$ are assumed to be independent, but not necessarily identically distributed.

By denoting the energy per pilot as $E_{P}$, the received signal at sensor $k$ during pilot transmission is

$$
r_{k}[n]=g_{k} \sqrt{E_{P}}+\eta_{k}[n] \quad n=1, \ldots, N_{P},
$$

where $\eta_{k}[n]$ is a complex Gaussian noise random variable (r.v.) with zero-mean and variance per dimension of $N_{0} / 2$. We now describe four models for transmission of the $N_{D}$ data bits. The four models have two things in common; the $N$ sensors cooperate to transmit their bits; and the schemes can be implemented in a completely distributed fashion, requiring no information exchange between sensors. The models primarily differ in the power at which they transmit their bits.

\section{A. Fixed Power Transmission Schemes}

We consider two transmission schemes in which the sensors transmit at a constant power whenever they transmit. This results in simpler and more efficient implementation of the transmitter RF circuitry. We analyze the performance of these two schemes separately in Sections III and IV for clarity of presentation.

1) Distributed Co-Phasing: In this model, we impose an instantaneous (or short-term) power constraint on the sensor node transmitters. Further, to reduce the modulator complexity we allow the sensors to compensate only for the channel phase. Thus, each sensor simply pre-rotates its bits with the estimated channel phase $\hat{\theta}_{k}$ and transmits the bits. With an average energy per modulation symbol of $E_{s}$ at each sensor, the received signal at the $\mathrm{FC}$ during $N_{D}$ data transmissions is

$$
r[n]=\sum_{k=1}^{N} x_{k}[n] e^{-j \hat{\theta}_{k}} g_{k}+V[n],
$$

where $n=N_{P}+1, \ldots, N_{P}+N_{D}, x_{k}[n]=b_{k} E_{s}, b_{k}$ is the modulation symbol at sensor $k, \hat{\theta}_{k}$ is the estimated downlink channel phase. Also, $x_{k}[n] e^{-j \hat{\theta}_{k}}$ is the actual transmitted signal from sensor $k$, and $V[n]$ is a zero-mean complex Gaussian r.v. with a per-dimension variance of $N_{0} / 2$. The model described in (2) is formally referred to as distributed co-phasing transmission.

2) Censoring sensors: In this scheme, the sensors are assumed to be aware of the channel statistics. They estimate the channel gain, and transmit only when the estimated channel gain is sufficiently good. We consider the two possibilities mentioned below; both options result in the same average received power at the FC. The average received power is made equal to that with the DCP described above, thus making the schemes directly comparable.

a) Case 1: In this scheme, referred to as CS-C1, we fix the number of sensors co-operating in the transmission, and adjust the transmit powers such that each sensor consumes the same average power as with DCP. The sensors transmit only when their estimated channel gain is above a threshold $T$. The sensors boost the power whenever they transmit, to ensure that each sensor satisfies an average power constraint. Further, similar to DCP, the sensors pre-rotate the transmitted bit with the estimated channel phase so that the symbols add up constructively at the FC. Note that, in this scheme, sensors use a fixed power (that depends on $T$ ) when they transmit, i.e., they do not employ dynamic power control.

With $x_{k}[n]$ as the transmitted bit at the sensor $k$, the received data signal at the $\mathrm{FC}$ is

$$
r[n]=\sum_{k=1}^{N} x_{k}[n] 1_{\left\{\hat{\alpha}_{k}>T\right\}} e^{-j \hat{\theta}_{k}} g_{k}+V[n],
$$

where $n=N_{P}+1, \ldots, N_{P}+N_{D}, x_{k}[n]=b_{k} \sqrt{\frac{E_{s}}{p_{T, k}}}$, $b_{k}= \pm 1$ is the modulation symbol at sensor $k, p_{T, k}$ is the probability that the sensor $k$ will transmit in a given time slot, $\hat{\alpha}_{k}$ is the estimated channel gain the sensor $k$. 
b) Case 2: In this scheme, referred to as CS-C2, the expected number of sensors that transmit at any given time is kept constant. We assume that the channels from all sensors to the FC have identical statistics. The total number of sensors which are deployed, denoted $N_{R}$, is varied to keep the expected number of transmitting sensors, denoted $N_{\text {eff }}$, a constant. All the sensors transmit with $E_{s}$ energy per modulation bit, thus keeping the average received power at the FC the same as in case 1 above.

Given a channel gain threshold of $T$, the number of sensors $N_{R}$ required for maintaining the average number of transmitting sensors as at least $N_{\text {eff }}$ is given by

$$
N_{R}=\left\lceil\frac{N_{e f f}}{\operatorname{Prob}\left(\hat{\alpha}_{k}>T\right)}\right\rceil
$$

The received signal at the $\mathrm{FC}$ is

$$
r[n]=\sum_{k=1}^{N_{R}} x_{k}[n] 1_{\left\{\hat{\alpha}_{k}>T\right\}} e^{-j \hat{\theta}_{k}} g_{k}+V[n],
$$

where $x_{k}[n]=b_{k} \sqrt{E_{s}}, b_{k}= \pm 1$ is a modulation symbol, $\hat{\alpha}_{k}$ and $\hat{\theta}_{k}$ are the estimated channel gain and channel phase, respectively, at the $k$-th sensor and in the current frame, and $g_{k}$ and $V[n]$ are defined as before.

\section{B. Variable Power Allocation Schemes}

We describe two schemes which employ power control at the transmitting sensors based on the estimated channel state. A long term transmit power constraint is imposed over the sensors and the transmit powers are chosen to satisfy this constraint.

1) Maximum Ratio Transmission (MRT): In this scheme, sensors transmit at a power that is proportional to the square of the estimated channel gain. The proportionality constant is chosen to satisfy an average power constraint at each sensor. The received signal at the FC thus has a form similar to the MRT scheme [22]. The power allocation scheme at sensor $i$ is given by

$$
P\left(\hat{\alpha}_{i}\right)=P_{0, i} \hat{\alpha}_{i}^{2}
$$

where $P_{0, i}$ is chosen to satisfy a long term average power constraint. The received signal at the fusion center is given by

$$
\begin{aligned}
y[n] & =\sum_{k=1}^{N} \sqrt{P\left(\hat{\alpha}_{k}\right)} b_{k} e^{-j \hat{\theta}_{k}} g_{k}+V[n] \\
& =\sum_{k=1}^{N} x_{k}[n] \hat{\alpha}_{k} e^{-j \theta_{e, k}} \alpha_{k}+V[n],
\end{aligned}
$$

where $b_{k}= \pm 1$ is the bit to be transmitted, $x_{k}=\sqrt{P_{0, i}} b_{k}$ is the modulation symbol from the sensor $k, \theta_{e, k}=\hat{\theta}_{k}-\theta_{k}$ is the phase estimation error, and $g_{k}, \hat{\theta}_{k}$ and $V[n]$ are as defined earlier.

2) Truncated Channel Inversion: In this model, the transmit power at sensor $i$ is given by [23]

$$
P\left(\hat{\alpha}_{i}\right)=\left\{\begin{aligned}
\frac{P_{0}}{\hat{\alpha}_{i}^{2}} & \text { if } \hat{\alpha}_{i}>\alpha_{\min }, \\
0 & \text { else }
\end{aligned}\right.
$$

where $P_{0}$ and $\alpha_{\min }$ are chosen to satisfy the long term average power constraint. The received signal at the FC is given by

$$
r[n]=\sum_{k=1}^{N} \sqrt{P\left(\hat{\alpha}_{k}\right)} b_{k} g_{k} e^{-j \hat{\theta}_{k}}+V[n],
$$

where $b_{k}= \pm 1$ is a modulation symbol from the sensor $i$, and $g_{k}, \hat{\theta}_{k}$ and $V[n]$ are defined as earlier.

\section{Performance Analysis of Distributed CO-PHASING}

The maximum likelihood estimate of the channel phase at sensor node $k$, using (1), is given by [24]

$$
\begin{aligned}
\hat{\theta}_{k} & =\tan ^{-1}\left(\frac{\Im\left\{\frac{1}{N_{P}} \sum_{n=1}^{N_{P}} r_{k}[n]\right\}}{\Re\left\{\frac{1}{N_{P}} \sum_{n=1}^{N_{P}} r_{k}[n]\right\}}\right) \\
& =\tan ^{-1}\left(\frac{\alpha_{k} \sin \theta_{k}+\nu_{Q, k}}{\alpha_{k} \cos \theta_{k}+\nu_{I, k}}\right),
\end{aligned}
$$

where $\nu_{I, k}$ and $\nu_{Q, k}$ are independent Gaussian r.vs each with zero mean and variance $\frac{N_{0}}{2 N_{P} E_{P}}$.

Conditioned on the channel gain $\alpha_{k}$, the cumulative distribution function of the phase error at node $k$ denoted by $\theta_{e, k} \triangleq \hat{\theta}_{k}-\theta_{k}$, can be expressed, following the simplifications in [25], as

$$
F_{\left|\theta_{e, k}\right| \mid \alpha_{k}}(x)=1-\frac{1}{\pi} \int_{0}^{\pi-x} e^{-\frac{\alpha_{k}^{2} \bar{\gamma}_{p} \sin ^{2} x}{\sin ^{2} \beta}} d \beta \quad 0 \leq x<\pi,
$$

where $\overline{\gamma_{p}}=E_{P} N_{P} / N_{0}$ is the pilot SNR.

\section{A. Distribution of Unconditional Phase Error}

Let the channel fade power at sensor node $k$ be denoted by $\gamma_{k} \triangleq \alpha_{k}^{2}$. We also denote the Laplace transform of the pdf of $\gamma_{k}$ as $\mathcal{L}_{\gamma_{k}}(s) \triangleq E\left[e^{-s \gamma_{k}}\right]$. With this notation, the unconditional distribution of the absolute phase error at node $k$ is obtained by integrating (11) over the distribution of $\gamma_{k}$ as

$$
\begin{aligned}
& F_{\left|\theta_{e, k}\right|}(x)=1-\frac{1}{\pi} \int_{0}^{\pi-x} E\left[e^{-\frac{\gamma_{k} \bar{\gamma}_{p} \sin ^{2} x}{\sin ^{2} \beta}}\right] d \beta \\
& =1-\int_{0}^{\pi-x} \mathcal{L}_{\gamma_{k}}\left(\frac{\bar{\gamma}_{p} \sin ^{2} x}{\sin ^{2} \beta}\right) \frac{d \beta}{\pi} \quad 0 \leq x<\pi .
\end{aligned}
$$

For a Rayleigh fading channel, $\mathcal{L}_{\gamma_{k}}(s)=1 /\left(1+s \Omega_{k}\right)$, where $\Omega_{k}=E\left[\alpha_{k}^{2}\right]$, whereas for a Rician fading channel with Rice factor $\mathrm{K}_{l}$, from [24], $\mathcal{L}_{\gamma_{k}}(s)=\left(1+\mathrm{K}_{l}\right) /(1+$ $\left.\mathrm{K}_{l}+s \Omega_{k}\right) e^{-s \mathrm{~K}_{l} \Omega_{k} /\left(1+\mathrm{K}_{l}+s \Omega_{k}\right)}$, and (12) can be evaluated accordingly.

\section{B. Probability of Signal Corruption}

The contribution of the channel gain from node $k$ to the FC in the uplink direction is $g_{k} e^{-j \hat{\theta}_{k}}$. Since we assume binary signaling in the uplink, the channel contribution to the decision variable is $\alpha_{k} \cos \left(\theta_{k}-\hat{\theta}_{k}\right)=\alpha_{k} \cos \theta_{e, k}$, which is negative when $\left|\theta_{e, k}\right|>\pi / 2$. That is, keeping the noise contribution 
at the FC aside, node $k$ causes signal corruption at the FC when $\left|\theta_{e, k}\right|>\pi / 2$. This probability is denoted by $P_{S C}$ and is obtained simply by using the CDF of $\theta_{e, k}$ in (12) as

$$
\begin{aligned}
P_{S C} & =\operatorname{Prob}\left(\left|\theta_{e, k}\right|>\pi / 2\right)=1-F_{\left|\theta_{e, k}\right|}(\pi / 2) \\
& =\frac{1}{\pi} \int_{0}^{\pi / 2} \mathcal{L}_{\gamma_{k}}\left(\frac{\bar{\gamma}_{p}}{\sin ^{2} \beta}\right) d \beta .
\end{aligned}
$$

For a Rayleigh fading channel, using $\mathcal{L}_{\gamma_{k}}(s)=\left(1+s \Omega_{k}\right)^{-1}$, the above evaluates to

$$
P_{S C}=\frac{1}{2}\left(1-\sqrt{\frac{\bar{\gamma}_{p} \Omega_{k}}{1+\bar{\gamma}_{p} \Omega_{k}}}\right) .
$$

Notice that $P_{S C}$ solely depends on the average received pilot SNR at the sensor nodes and the channel fade statistics, and at high uplink data SNR, signal corruption has a dominant effect on the detection performance at the FC.

To calculate the average received SNR and BER at the FC, we consider two cases. When the sensors can detect the common bit that they wish to coney to the fusion center perfectly, all the sensors have the same bit to transmit. When the sensors make imperfect decisions, they may have different bits to transmit based on their local decisions. We assume conditionally independent observations at the sensors, and that the individual probabilities of missed detection and false alarm $^{1}$ are known and the same at all sensors. This models a distributed detection scenario, where a FC uses the individual decisions from a set of nodes to arrive at an overall decision. Here, we are effectively performing a physical layer fusion of the individual sensor decisions, at the FC.

\section{Sensors with Perfect Detection}

We now find the average received SNR and the average probability of error when all the sensors have perfect detection; i.e., they have the same bit to send to the FC.

1) Average Received SNR: The test statistic at the FC, from (2), is $^{2}$

$$
\begin{aligned}
r[n] & =\Re\left\{\sum_{k=1}^{N} x[n] g_{k} e^{-j \hat{\theta}_{k}}+V[n]\right\} \\
& =x[n] \sum_{k=1}^{N} \alpha_{k} \cos \theta_{e, k}+V_{r}[n],
\end{aligned}
$$

where $V_{r}[n]$ is a real Gaussian random variable with zero mean and variance $N_{0} / 2$. Assuming spatial independence of the channels across the sensors, the average received SNR at

\footnotetext{
${ }^{1} \mathrm{~A}$ missed detection occurs when a sensor detects a binary ' 1 ' as a ' 0 ', and a false alarm occurs when it detects a binary ' 0 ' as a ' 1 '.

${ }^{2}$ It is straightforward to show that under our system model, the real part of the signal is sufficient for BPSK signal detection, since the sensors do not transmit any training symbols to the fusion center.
}

the FC is

$$
\begin{aligned}
\bar{\gamma}_{D C P}= & \frac{2 E_{s}}{N_{0}} E\left[\left|\sum_{k=1}^{N} \alpha_{k} \cos \theta_{e, k}\right|^{2}\right] \\
= & \frac{2 E_{s}}{N_{0}}\left[\sum_{k=1}^{N} E\left[\alpha_{k}^{2} \cos ^{2} \theta_{e, k}\right]+\sum_{k=1}^{N} \sum_{j=1, j \neq k}^{N}\right. \\
& \left.E\left[\alpha_{k} \cos \theta_{e, k}\right] E\left[\alpha_{j} \cos \theta_{e, j}\right]\right]
\end{aligned}
$$

For Rayleigh fading channels, using (61) from Appendix-A, we have

$$
E\left[\alpha_{k} \cos \theta_{e, k}\right]=\sqrt{\frac{\pi \Omega_{k}}{4}} \sqrt{\frac{\bar{\gamma}_{p} \Omega_{k}}{1+\bar{\gamma}_{p} \Omega_{k}}},
$$

and, using (66) from Appendix-B,

$$
E\left[\alpha_{k}^{2} \cos ^{2} \theta_{e, k}\right]=\Omega_{k}\left[1-\frac{1}{2}\left(\frac{1}{1+\bar{\gamma}_{p} \Omega_{k}}\right)\right] .
$$

Closed-form expressions for $\bar{\gamma}_{D C P}$ can then be obtained by using (17) and (18) in (16).

2) Average Probability of Error: Let the sensors transmit the symbol $x[n]=\sqrt{E_{s}}$ corresponding to the binary digit ' 1 '. Then, the decision variable (15) at the FC is

$$
r[n]=\sqrt{E_{s}} \sum_{k=1}^{N} \alpha_{k} \cos \theta_{e, k}+V_{r}[n] .
$$

With equal prior probabilities on the bits, the $\mathrm{FC}$ makes an error when $r[n]<0$. An exact expression for the average BER can therefore be obtained by averaging the conditional probability of error

$$
P_{e}\left(\alpha_{1}, \theta_{e, 1}, \ldots, \alpha_{N}, \theta_{e, N}\right)=\mathcal{Q}\left(\sqrt{\frac{2 E_{s}}{N_{0}}} \sum_{k=1}^{N} \alpha_{k} \cos \theta_{e, k}\right)
$$

over the joint distribution of $\left(\alpha_{k}, \theta_{e, k}\right), k=1, \ldots, N$. However, this is a difficult task to perform even for a small value of $N$. On the other hand, when the size $N$ of the participating sensor nodes is reasonably large we can simplify (20) by applying a Gaussian approximation to the argument in (20). In this paper, we use the Improved Gaussian Approximation (IGA) technique suggested in [20]. As per [20], by defining $R=\sqrt{2 E_{s} / N_{0}} \sum_{k=1}^{N} \alpha_{k} \cos \theta_{e, k}$, an approximation to the average of (20) is

$$
\begin{aligned}
\bar{P}_{e} \approx & \frac{2}{3} \mathcal{Q}(E[R])+\frac{1}{6} \mathcal{Q}\left(E[R]+\sqrt{3} \sqrt{E\left[R^{2}\right]-E[R]^{2}}\right) \\
& +\frac{1}{6} \mathcal{Q}\left(E[R]-\sqrt{3} \sqrt{E\left[R^{2}\right]-E[R]^{2}}\right)
\end{aligned}
$$

where, using (17) and (18),

$$
\begin{aligned}
E[R]= & \sqrt{\frac{2 E_{s}}{N_{0}}} \sum_{k=1}^{N} \sqrt{\frac{\pi \Omega_{k}}{4}} \sqrt{\frac{\bar{\gamma}_{p} \Omega_{k}}{1+\bar{\gamma}_{p} \Omega_{k}}} \\
\text { and } E\left[R^{2}\right]= & \frac{2 E_{s}}{N_{0}} \sum_{k=1}^{N} \Omega_{k}\left[\frac{1+2 \bar{\gamma}_{p} \Omega_{k}}{2\left(1+\bar{\gamma}_{p} \Omega_{k}\right)}\right]+\frac{\pi E_{s}}{2 N_{0}} \times \\
& \sum_{k=1}^{N} \sum_{j=1, j \neq k}^{N} \sqrt{\frac{\bar{\gamma}_{p} \Omega_{k}^{2}}{1+\bar{\gamma}_{p} \Omega_{k}}} \sqrt{\frac{\bar{\gamma}_{p} \Omega_{j}^{2}}{1+\bar{\gamma}_{p} \Omega_{j}}}(23)
\end{aligned}
$$




\section{Sensors with Imperfect Detection}

Now consider the case when the sensors trying to detect an underlying random variable using conditionally independent observations. Suppose that the sensors decide between two hypothesis $H_{0}$, represented by the bit ' 0 ', and $H_{1}$, represented by the bit ' 1 ' and send their decision to the FC. Let all sensors have the same probability of false alarm, $P_{F A}$, and probability of miss detection, $P_{M}$.

Assume that $H_{0}$ is the true hypothesis; the analysis when $H_{1}$ is true is similar. Let $\underline{b} \in \mathcal{D}^{N}$ be the vector of observations transmitted by the $N$ sensors, where $\mathcal{D}=\{-1,1\}$. Sensors that decide in favor of $H_{0}$ transmit a ' -1 ', and sensors that make a false alarm transmit a ' +1 '. Let $K$ be the number of false alarms out of these $N$ sensors. Assuming identical false alarm rates, we have $P(\underline{b})=\left(1-P_{F A}\right)^{N-K} P_{F A}^{K}$. With this, the average received SNR and the average BER can be derived as follows.

1) Average Received SNR: Starting from (2) with $x_{k}[n]=$ $b_{k} \sqrt{E_{s}}$, and following (15) and (16), we have

$$
\begin{aligned}
\bar{\gamma}_{D C P}= & \frac{2 E_{s}}{N_{0}} E\left\{\left|\sum_{k=1}^{N} b_{k} \alpha_{k} \cos \theta_{e, k}\right|^{2}\right\} \\
= & \frac{2 E_{s}}{N_{0}}\left\{\sum_{k=1}^{N} E\left[\alpha_{k}^{2} \cos ^{2} \theta_{e, k}\right] E\left[b_{k}^{2}\right]+\right. \\
& \left.\sum_{i=1}^{N} \sum_{j=1, i \neq j}^{N} E\left[\alpha_{i} \cos \theta_{e, i}\right] E\left[\alpha_{j} \cos \theta_{e, j}\right] E\left[b_{i} b_{j}\right]\right\} .
\end{aligned}
$$

Note that the difference between (16) and the above equation is that in the former, since identical data is sent by the sensors, $x_{k}[n]=x[n]$ comes out of the summation and is incorporated into the $\frac{2 E_{s}}{N_{0}}$ coefficient. Also, (24) requires that $b_{i}$ be independent of the channel between the sensors and the FC, which is a reasonable assumption. Using $E\left[b_{k}^{2}\right]=1$, $E\left[b_{i} b_{j}\right]=\left(1-2 P_{F A}\right)^{2}$ for $i \neq j, E\left[\alpha_{i} \cos \theta_{e, i}\right]$ from (17), and $E\left[\alpha_{k}^{2} \cos ^{2} \theta_{e, k}\right]$ from (18), a closed form expression for $\bar{\gamma}_{D C P}$ can be obtained, similar to the previous subsection. The details are omitted due to lack of space.

2) Average Probability of Error: We start from (2) with $x_{k}[n]=b_{k} \sqrt{E_{s}}$ and follow a procedure similar to the previous subsection. When $H_{0}$ is true, an error occurs in the decision if $r[n]>0$, i.e., if $V_{r}[n]>-\sqrt{E_{s}} \sum_{k=1}^{N} b_{k} \alpha_{k} \cos \theta_{e, k}$. The average BER is then

$$
\begin{aligned}
& P_{e}\left(b_{1}, \alpha_{1}, \theta_{e, 1}, \ldots b_{N}, \alpha_{N}, \theta_{e, N}\right)= \\
& 1-\mathcal{Q}\left(\sqrt{\frac{2 E_{s}}{N_{0}}} \sum_{l=1}^{N} b_{l} \alpha_{l} \cos \theta_{e, l}\right) .
\end{aligned}
$$

An exact expression for the average BER can be obtained by averaging (25) over the joint distribution of $\left(b_{k}, \alpha_{k}, \theta_{e, k}\right), k=$ $1, \ldots, N$. To simplify the analysis, we further assume that the statistics of the individual channels from the FC to the sensors are i.i.d. Then, for $i=1,2, \ldots, N, E\left[\alpha_{i}{ }^{2} \cos ^{2} \theta_{e, i}\right]=$

$$
\begin{gathered}
E\left[\alpha^{2} \cos ^{2} \theta_{e}\right], E\left[\alpha_{i} \cos \theta_{e, i}\right]=E\left[\alpha \cos \theta_{e}\right], \text { and } \\
P_{e}=\sum_{K=0}^{N}\left(\begin{array}{l}
N \\
K
\end{array}\right)\left(1-P_{F A}\right)^{N-K} P_{F A}^{K} \\
\left(1-E_{\underline{\theta_{e}}, \underline{\alpha} \mid \underline{b}}\left(\mathcal{Q}\left(\sqrt{\frac{2 E_{s}}{N_{0}}} \sum_{l=1}^{N} b_{l} \alpha_{l} \cos \theta_{e, l}\right)\right)\right)
\end{gathered}
$$

where $K$ is the number of errors in the vector $\underline{b}$ and $b_{l}$ thus depends on the value of is now actually a function of $K$. Upon defining $R_{1}=\sqrt{\frac{2 E_{s}}{N_{0}}} \sum_{l=1}^{N} b_{l} \alpha_{l} \cos \theta_{e, l}$, and using the IGA for obtaining $E_{\theta_{e}, \underline{\alpha} \mid \underline{b}}\left[\mathcal{Q}\left(R_{1}\right)\right]$, we have (34) and (35). Note that (35) follows from the fact that the number of ' -1 's and ' +1 's in $\underline{b}$ are $K$ and $(N-K)$ respectively. The average probability of error can now be obtained by substituting (35) in (34) and substituting the resulting expression in (26). Note that $E\left[\alpha \cos \theta_{e}\right]$ and $E\left[\alpha^{2} \cos ^{2} \theta_{e}\right]$ are given by (17) and (18) for a Rayleigh fading channel. Note also that the probability of error in (26) is conditional on $H_{0}$ being true, i.e., it is the overall probability of false alarm.

If the probability of miss detection is represented by $P_{M}$, then when $H_{1}$ is true, the average overall probability of missed detection can be similarly derived to be

$$
\begin{aligned}
P_{e}= & \sum_{K=0}^{N}\left(\begin{array}{l}
N \\
K
\end{array}\right)\left(1-P_{M}\right)^{N-K} P_{M}{ }^{K} \\
& \mathbb{E}_{\underline{\theta_{e}}, \underline{\alpha}}\left(\mathcal{Q}\left(\sqrt{\frac{2 E_{s}}{N_{0}}} \sum_{l=1}^{N} b_{l} \alpha_{l} \cos \theta_{e, l}\right)\right) .
\end{aligned}
$$

Closed-form expressions for the expectation term above can be derived using the IGA in a similar manner as above. The details are omitted here to avoid repetition.

\section{Performance AnAlysis of Censoring SEnsors}

We shall now analyze two schemes, CS-C1 and CS-C2, in which the sensors are constrained to transmit only when the channel is sufficiently good. The intuition behind such schemes is that sensors that are in a bad channel state contribute very little to the overall decision. So, the question is, can one not improve the performance if the sensors save their power when the channel state is bad, and transmit at a higher power (or, if larger number of sensors are deployed to maintain the same total transmit power) compared to the non-censoring case when the channel state is good? Both schemes considered here are distributed, and are comparable to the previous section in the sense that the total average transmit power across the sensors is maintained the same as with no censoring. In the first scheme (CS-C1), the sensors transmit only when the estimated channel gain is above a certain threshold. However, when they do transmit, they boost their power by the inverse of the probability of transmission to make the average transmit power per sensor the same as in the non-censoring case. In the second scheme (CS-C2), the sensors again transmit only when the estimated channel gain exceeds a threshold, but the number of sensors are increased so that, on an average, the same number of sensors transmit as in the non-censoring case. Here, the transmit power is not boosted since on an average 
$N$ of these sensors will transmit keeping the average total power the same as in the non-censoring case. Also, both schemes employ a fixed transmit power at the sensors as long as the channel statistics remain unchanged. The performances of these two schemes in terms of average received SNR at the FC are derived below.

\section{A. CS-C1: Fixed number of sensors with power boosting}

The same frame structure as in the non-censoring case is considered here. With $N_{P}$ pilot symbols, the complex-valued channel estimate at sensor $k$ is

$\hat{g}_{k}=\frac{1}{N_{P} \sqrt{E_{P}}} \sum_{n=1}^{N_{P}} r_{k}[n]=g_{k}+\frac{1}{N_{P} \sqrt{E_{P}}} \sum_{n=1}^{N_{P}} \eta_{k}[n]=g_{k}+\nu_{k}$,

where $r_{k}[n]=g_{k}+\eta_{k}[n]$ and $\nu_{k} \sim \mathcal{C N}\left(0, N_{0} / E_{P} N_{P}\right)$. Writing $g_{k}=\alpha_{k} e^{j \theta_{k}}, \hat{g_{k}}=\hat{\alpha}_{k} e^{j \hat{\theta}_{k}}$, and pre-multiplying (28) by $e^{-j \theta_{k}}$, we have $\hat{\alpha}_{k} e^{j\left(\hat{\theta}_{k}-\theta_{k}\right)}=\alpha_{k}+\nu_{k} e^{-j \theta_{k}}$. Note that $\nu_{k} e^{-j \theta_{k}}$ and $\nu_{k}$ have the same distribution, and $\nu_{k} e^{-j \theta_{k}}$ and $\alpha_{k}$ are independent. Let $w_{k}=\nu_{k} e^{-j \theta_{k}}=w_{1}^{k}+j w_{2}^{k}$, $\theta_{e, k}=\hat{\theta}_{k}-\theta_{k}$, and $\hat{\alpha}_{k} e^{j \theta_{e, k}}=X_{1}^{k}+j X_{2}^{k}$. Then $X_{1}^{k}=$ $\alpha_{k}+w_{1}^{k}, X_{2}^{k}=w_{2}^{k}$, and $w_{1}^{k}, w_{2}^{k} \sim \mathcal{N}\left(0, \frac{N_{0}}{2 E_{P} N_{P}}\right)$. Writing $\sigma^{2} \triangleq \frac{N_{0}}{2 N_{P}}$, we have, using a polar coordinate transformation of $X_{1}^{k}=\hat{\alpha}_{k} \cos \theta_{e, k}$ and $X_{2}^{k}=\hat{\alpha}_{k} \sin \theta_{e, k}$,

$$
f_{\hat{\alpha}_{k}, \theta_{e, k} \mid \alpha_{k}}(R, \theta)=\frac{R}{2 \pi \sigma^{2}} e^{-\frac{R^{2}-2 R \alpha_{k} \cos \theta+\alpha_{k}^{2}}{2 \sigma^{2}}} .
$$

With a transmission policy that sensor $k$ will transmit only if its estimated channel gain is above a certain threshold $T$, the decision variable at the $\mathrm{FC}$ is

$$
\begin{aligned}
z[n] & =\sum_{k=1}^{N} x_{k}[n] \underbrace{1_{\left\{\hat{\alpha}_{k}>T\right\}} \alpha_{k} \cos \theta_{e, k}}_{\triangleq z_{k}}+\underbrace{\eta[n]}_{\sim \mathcal{C N}\left(0, \sigma_{N}^{2}\right)}, \\
& =\sum_{k=1}^{N} x_{k} z_{k}+\eta,
\end{aligned}
$$

where $x_{k}=b_{k} \sqrt{E_{s, k}}$ is the modulation symbol with $b_{k}= \pm 1$ and $E_{s, k}$ is the energy per symbol transmitted at the sensor $k, \sigma_{N}^{2}$ is the variance of the noise $\eta[n]$ added at the $\mathrm{FC}$, and the time index is dropped since it does not affect the analysis that follows. Thus, the average received SNR, $\bar{\gamma}_{C S, 1}$, is

$$
\begin{aligned}
\bar{\gamma}_{C S-C 1}= & \frac{1}{\sigma_{N}^{2}} E\left[\sum_{k=1}^{N} E_{s, k} b_{k}^{2} z_{k}^{2}+\right. \\
& \left.\sum_{k=1}^{N} \sum_{j=1, k \neq j}^{N} \sqrt{E_{s, k} E_{s, j}} b_{k} b_{j} z_{k} z_{j}\right] .
\end{aligned}
$$

Since the sensor $k$ transmits only when the estimated channel gain $\hat{\alpha}_{k}$ is above the threshold $T$, the energy per modulation symbol is scaled such that the average power constraint on that sensor is satisfied. That is, $E_{s, k}=E_{s} / p_{T, k}$, where $p_{T, k}=$ $\operatorname{Prob}\left\{\hat{\alpha}_{k}>T\right\}$ is the probability of transmission at sensor $k$.
For Rayleigh fading channels,

$$
\begin{aligned}
\operatorname{Prob}\left\{\hat{\alpha}_{k}>T\right\} & =\int_{T}^{\infty} f_{\hat{\alpha}_{k}}(x) d x=\int_{T}^{\infty} \frac{2 x e^{-\frac{x^{2}}{\Omega_{k}+2 \sigma^{2}}} d x}{\Omega_{k}+2 \sigma^{2}} \\
& =\exp \left(-\frac{T^{2}}{\Omega_{k}+2 \sigma^{2}}\right) .
\end{aligned}
$$

Then, we have

$$
\begin{aligned}
\bar{\gamma}_{C S-C 1}= & \sum_{k=1}^{N} \frac{E_{s} E\left[z_{k}^{2}\right]}{\sigma_{N}^{2} p_{T, k}}+ \\
& \sum_{k=1}^{N} \sum_{j=1, j \neq k}^{N} \frac{E_{s} E\left[b_{k} b_{j}\right] E\left[z_{k}\right] E\left[z_{j}\right]}{\sigma_{N}^{2} \sqrt{p_{T, k} p_{T, j}}} .
\end{aligned}
$$

The first and second moments of $z_{k}$ for the Rayleigh fading channel are evaluated in Appendix-C and are obtained from (46) and (47) as shown in (36) and (37), where $\Gamma(x, n)=$ $\int^{\infty} e^{-u} u^{n-1} d u / \Gamma(n)$ is the incomplete Gamma function [26]. $\stackrel{x}{A}$ closed form expression for average received SNR at the FC can now be obtained by using (36) and (37) in (33).

\section{B. CS-C2: Variable number of fixed-power sensors}

Substituting (32) in (4), the required number of sensors with Rayleigh fading channels, a transmission threshold of $T$, and the channel estimation scheme as in (28) is $N_{R}=$ $\left[N_{e f f} \exp \left(\frac{T^{2}}{\Omega_{k}+2 \sigma^{2}}\right)\right]$, where $N_{\text {eff }}$ is the average number of sensors which transmit.

The average received SNR at the FC has a similar expression as (33):

$$
\begin{aligned}
\bar{\gamma}_{C S-C 2}= & \frac{E_{s}}{\sigma_{N}{ }^{2}}\left[\sum_{k=1}^{N_{R}} E\left[z_{k}^{2}\right]+\right. \\
& \left.\sum_{k=1}^{N_{R}} \sum_{j=1, j \neq k}^{N_{R}} E\left[b_{k} b_{j}\right] E\left[z_{k}\right] E\left[z_{j}\right]\right] .
\end{aligned}
$$

\section{Performance Analysis of Variable Power ALLOCATION SCHEMES}

We now analyze the performance of the two variable power transmission schemes described in Section II-B, in which the sensors transmit at a power that is a function of the estimated channel gain. The schemes described here are distributed and the average transmit power at the sensors is maintained the same as the (constant) instantaneous transmit power in the DCP scheme. In the first scheme (MRT), the transmit power is proportional to the square of the estimated channel gain. In the second scheme, the transmit power is inversely proportional to the square of the estimated channel gain. At first glance, one would expect the variable power allocation schemes to perform better than the DCP scheme. This is because they use both the estimated gain and phase of the channel to manage their available power more efficiently. In MRT, the power is managed such that sensors transmit with higher power when their gains to the fusion center are higher. In TCI, the sensors ensure that if they transmit, it is such that their decisions arrive at the FC with equal power, which is desirable. Interestingly, 


$$
\begin{aligned}
& E_{\underline{\theta}_{e}, \underline{\alpha} \mid \underline{b}}\left[\mathcal{Q}\left(R_{1}\right)\right] \approx \frac{2}{3} \mathcal{Q}\left(E\left[R_{1}\right]\right)+\frac{1}{6} \mathcal{Q}\left(E\left[R_{1}\right]+\sqrt{3} \sqrt{E\left[R_{1}^{2}\right]-E\left[R_{1}\right]^{2}}\right)+\frac{1}{6} \mathcal{Q}\left(E\left[R_{1}\right]-\sqrt{3} \sqrt{E\left[R_{1}^{2}\right]-E\left[R_{1}\right]^{2}}\right), \\
& \text { where } E_{\underline{\theta_{e}}, \underline{\alpha} \mid \underline{b}}\left[R_{1}\right]=\sqrt{\frac{2 E_{s}}{N_{0}}}(2 K-N) E\left[\alpha \cos \theta_{e}\right], \quad \text { and } \quad E_{\underline{\theta_{e}}, \underline{\alpha} \mid \underline{b}}\left[R_{1}^{2}\right]=\frac{2 E_{s}}{N_{0}}\left(N E\left[\alpha^{2} \cos ^{2} \theta_{e}\right]+\left((2 K-N)^{2}-N\right) E\left[\alpha \cos \theta_{e}\right]^{2}\right) .
\end{aligned}
$$

$$
\begin{aligned}
E\left[z_{k}\right] & =\sqrt{\Omega_{k}} \sum_{\ell=0}^{\infty} \frac{\Gamma\left(\ell+\frac{3}{2}\right) \Gamma\left(\frac{T^{2}}{2 \sigma^{2}}, \ell+\frac{3}{2}\right)}{\ell !}\left(\frac{1}{1+\frac{\Omega_{k}}{2 \sigma^{2}}}\right)^{\ell+2}\left(\frac{\Omega_{k}}{2 \sigma^{2}}\right)^{\ell+\frac{1}{2}}, \\
E\left[z_{k}^{2}\right] & =\Omega_{k} \sum_{\ell=0}^{\infty} \frac{1}{2} \frac{\Gamma(l+2)}{\ell !}\left(\frac{\Omega_{k}}{2 \sigma^{2}}\right)^{\ell}\left(\frac{1}{1+\frac{\Omega_{k}}{2 \sigma^{2}}}\right)^{\ell+2}\left[\Gamma\left(\frac{T^{2}}{2 \sigma^{2}}, \ell+1\right)+\left(\frac{\Omega_{k}}{2 \sigma^{2}}\right)\left(\frac{1}{1+\frac{\Omega_{k}}{2 \sigma^{2}}}\right) \Gamma\left(\frac{T^{2}}{2 \sigma^{2}}, \ell+2\right)\right] .
\end{aligned}
$$

$$
\begin{aligned}
\gamma_{M R T} & =\frac{1}{\sigma_{N}^{2}}\left[\sum_{k=1}^{N} E\left[x_{k}^{2}\right]\left(\frac{\Omega_{k}^{2}}{2}\left(4+\frac{1}{\frac{\Omega_{k}}{2 \sigma^{2}}}\right)\right)+\sum_{j=1}^{N} \sum_{i=1, i \neq j}^{N} E\left[x_{i} x_{j}\right] \Omega_{i} \Omega_{j}\right] \\
& =\frac{1}{\sigma_{N}^{2}}\left[\sum_{k=1}^{N} P_{0, k} E\left[b_{k}^{2}\right]\left(\frac{\Omega_{k}^{2}}{2}\left(4+\frac{1}{\frac{\Omega_{k}}{2 \sigma^{2}}}\right)\right)+\sum_{j=1}^{N} \sum_{i=1, i \neq j}^{N} E\left[b_{i} b_{j}\right] \sqrt{P_{0, i} P_{0, j} \Omega_{i} \Omega_{j}}\right] \\
& \left.=\frac{\bar{P}}{\sigma_{N}^{2}}\left[\sum_{k=1}^{N} \frac{\Omega_{k}}{2}\left(\frac{1+\frac{4 \Omega_{k}}{2 \sigma^{2}}}{1+\frac{\Omega_{k}}{2 \sigma^{2}}}\right)+\sum_{j=1}^{N} \sum_{i=1, i \neq j}^{N} E\left[b_{i} b_{j}\right] \sqrt{\Omega_{i} \Omega_{j}} \sqrt{\left(\frac{\Omega_{i}}{2 \sigma^{2}}\right.} \frac{\frac{\Omega_{j}}{2 \sigma_{i}^{2}}}{2 \sigma^{2}}\right)\right] .
\end{aligned}
$$

$$
\begin{aligned}
\bar{\gamma}_{T C I} & =\frac{1}{\sigma_{N}^{2}} E\left[\left|\sum_{i=1}^{N} \sqrt{P\left(\hat{\alpha}_{i}\right)} b_{i} \alpha_{i} \cos \theta_{e, i}\right|^{2}\right] \\
& =\frac{1}{\sigma_{N}^{2}}\left[\sum_{i=1}^{N} E\left[P\left(\hat{\alpha}_{i}\right) \alpha_{i}{ }^{2} \cos ^{2} \theta_{e, i}\right]+\sum_{i=1}^{N} \sum_{j=1, j \neq i}^{N} E\left[b_{i} b_{j}\right] E\left[\sqrt{P\left(\hat{\alpha}_{i}\right)} \alpha_{i} \cos \theta_{e, i}\right] E\left[\sqrt{P\left(\hat{\alpha}_{j}\right)} \alpha_{j} \cos \theta_{e, j}\right]\right]
\end{aligned}
$$

$$
\begin{aligned}
E\left[\alpha_{k} \cos \theta_{e, k}\right] & =\sqrt{\frac{\bar{\gamma}_{p}}{4 \pi}} \int_{-1}^{+1} \frac{1}{\sqrt{1-t^{2}}} E\left[\gamma_{k} e^{-\gamma_{k} s(1+t)}\right] d t+s \sqrt{\frac{\bar{\gamma}_{p}}{4 \pi}} \int_{-1}^{+1} \sqrt{1-t^{2}} E\left[\gamma_{k}^{2} e^{-\gamma_{k} s(1+t)}\right] d t \\
& =-\left.\frac{1}{2} \sqrt{\frac{\bar{\gamma}_{p}}{\pi}} \int_{-1}^{+1} \frac{d t}{\sqrt{1-t^{2}}} \frac{d \mathcal{L}_{\gamma_{k}}(u)}{d u}\right|_{u=s(1+t)}+\left.\frac{\bar{\gamma}_{p}}{4} \sqrt{\frac{\bar{\gamma}_{p}}{\pi}} \int_{-1}^{+1} \sqrt{1-t^{2}} \frac{d^{2} \mathcal{L}_{\gamma_{k}}(u)}{d u^{2}}\right|_{u=s(1+t)} d t .
\end{aligned}
$$

$$
E\left[\alpha_{k} \cos \theta_{e, k}\right]=\frac{\sqrt{\Omega_{k}}}{2} \sqrt{\frac{\bar{\gamma}_{p} \Omega_{k}}{\pi}} \int_{-1}^{+1} \frac{1}{\sqrt{1-t^{2}}}\left(\frac{1}{1+s \Omega_{k}(1+t)}\right)^{2} d t+\frac{\bar{\gamma}_{p} \Omega_{k} \sqrt{\Omega_{k}}}{4} \sqrt{\frac{\bar{\gamma}_{p} \Omega_{k}}{\pi}} \int_{-1}^{+1} \sqrt{1-t^{2}}\left(\frac{2}{\left(1+s \Omega_{k}(1+t)\right)^{3}}\right) d t
$$

$$
\begin{aligned}
\mathcal{I}\left(\alpha_{k}\right)= & \frac{\bar{\gamma}_{p} \alpha_{k}^{2}}{\pi} \int_{x=0}^{\pi} \frac{1}{\sin ^{2} x} \int_{\theta=0}^{\pi-x} \cos 2 \theta \sin 2 \theta e^{-\frac{\bar{\gamma}_{p} \alpha_{k}^{2} \sin ^{2} \theta}{\sin ^{2} x}} d \theta d x=\frac{\bar{\gamma}_{p} \alpha_{k}^{2}}{\pi} \int_{x=0}^{\pi} \frac{1}{\sin ^{2} x} \int_{t=0}^{\sin ^{2} x}(1-2 t) e^{-\frac{\bar{\gamma}_{p} \alpha_{k}^{2} t}{\sin ^{2} x} d t d x \quad(\text { with }} d t \int_{x=0}^{\pi} \frac{1}{\bar{\gamma}_{p} \alpha_{k}^{2}}\left[\bar{\gamma}_{p} \alpha_{k}^{2}\left(1-e^{-\bar{\gamma}_{p} \alpha_{k}^{2}}\right)+2\left(e^{-\bar{\gamma}_{p} \alpha_{k}^{2}}+\bar{\gamma}_{p} \alpha_{k}^{2} e^{-\bar{\gamma}_{p} \alpha_{k}^{2}}-1\right) \sin ^{2} x\right] d x=1-\frac{1-e^{-\bar{\gamma}_{p} \alpha_{k}^{2}}}{\bar{\gamma}_{p} \alpha_{k}^{2}} \\
= & \frac{1}{m_{k}(n)}=\int_{\alpha_{k}=0}^{\infty} \int_{\hat{\alpha}_{k}=0}^{\infty} \int_{\theta_{e, k}=-\pi}^{\pi} \alpha_{k}^{n}\left(1_{\left\{\hat{\alpha}_{k}>T\right\}}\right)^{n} \cos ^{n} \theta_{e, k} f_{\alpha_{k}}\left(\alpha_{k}\right) f_{\hat{\alpha}_{k}, \theta_{e, k} \mid \alpha_{k}}\left(\hat{\alpha}_{k}, \theta_{e, k}\right) d \hat{\alpha}_{k} d \alpha_{k} d \theta \\
= & \int_{\alpha_{k}=0}^{\infty} \alpha_{k}^{n} f_{\alpha_{k}}\left(\alpha_{k}\right)\left[\int_{\hat{\alpha}_{k}=T}^{\infty}\left[\int_{\theta=-\pi}^{\pi} \cos ^{n} \theta f_{\hat{\alpha}_{k}, \theta_{e, k} \mid \alpha_{k}}\left(\hat{\alpha}_{k}, \theta\right) d \theta\right] d \hat{\alpha}_{k}\right] d \alpha_{k} .
\end{aligned}
$$

it will turn out that the MRT scheme outperforms the DCP scheme, which outperforms the TCI scheme. We now derive expressions for the average received SNR at the FC for these two schemes.

\section{A. Maximum Ratio Transmission}

The power allocation at a given sensor is given by (6). The sensors are constrained by an average power $\bar{P}$, i.e., $E\left[\left|\sqrt{P_{0, k}} \hat{\alpha}_{k}\right|^{2}\right]=\bar{P}$ at sensor $k$. Thus the sensor power level $P_{0, k}$ is given by

$$
P_{0, k}=\frac{\bar{P}}{\Omega_{k}+2 \sigma^{2}}
$$




$$
\begin{aligned}
S\left(\alpha_{k}, 1, T\right) & =e^{-\frac{\alpha_{k}^{2}}{2 \sigma^{2}}} \int_{\hat{\alpha}_{k}=T}^{\infty} \frac{x}{\sigma^{2}} e^{-\frac{x^{2}}{2 \sigma^{2}}} \sum_{\ell=0}^{\infty} \frac{x^{2 \ell+1} \alpha_{k}^{2 \ell+1}}{\left(\sigma^{2}\right)^{2 \ell+1} 2^{2 \ell+1} \ell !(\ell+1) !} d x=e^{-\frac{\alpha_{k}^{2}}{2 \sigma^{2}}} \sum_{\ell=0}^{\infty} \frac{\alpha_{k}^{2 \ell+1}}{2^{2 \ell+1} \ell !(\ell+1) !} \int_{\frac{T^{2}}{2 \sigma^{2}}}^{\infty} \frac{e^{-y}\left(\sqrt{2 y \sigma^{2}}\right)^{2 \ell+1}}{\left(\sigma^{2}\right)^{2 \ell+1}} d y \\
& =e^{-\frac{\alpha_{k}^{2}}{2 \sigma^{2}}} \sum_{\ell=0}^{\infty} \frac{\alpha_{k}^{2 \ell+1}}{\left(2 \sigma^{2}\right)^{\frac{2 \ell+1}{2}} \ell !(\ell+1) !} \times \Gamma\left(\frac{T^{2}}{2 \sigma^{2}}, \frac{2 \ell+3}{2}\right) \Gamma\left(\frac{2 \ell+3}{2}\right), \\
S\left(\alpha_{k}, 2, T\right) & =\int_{\hat{\alpha}_{k}}^{\infty} \frac{\hat{\alpha}_{k}}{2 \sigma^{2}} e^{-\frac{\hat{\alpha}_{k}^{2}+\alpha_{k}^{2}}{2 \sigma^{2}}}\left[I_{0}\left(\frac{\hat{\alpha}_{k} \alpha_{k}}{\sigma^{2}}\right)+I_{2}\left(\frac{\hat{\alpha}_{k} \alpha_{k}}{\sigma^{2}}\right)\right] d \hat{\alpha}_{k} \\
& =e^{-\frac{\alpha_{k}^{2}}{2 \sigma^{2}}} \sum_{\ell=0}^{\infty} \frac{1}{2} \frac{\left(\alpha_{k}\right)^{2 \ell}}{\ell !\left(2 \sigma^{2}\right)^{\ell}} \Gamma\left(\frac{T^{2}}{2 \sigma^{2}}, \ell+1\right)+e^{-\frac{\alpha_{k}^{2}}{2 \sigma^{2}}} \sum_{\ell=0}^{\infty} \frac{1}{2} \frac{\left(\alpha_{k}\right)^{2 \ell+2}}{(\ell+2) ! \ell !\left(2 \sigma^{2}\right)^{\ell+1}} \Gamma\left(\frac{T^{2}}{2 \sigma^{2}}, \ell+2\right) \Gamma(\ell+2) .
\end{aligned}
$$

$$
\begin{aligned}
E\left[z_{k}\right] & =\int_{\alpha_{k}=0}^{\infty} \alpha_{k} f_{\alpha_{k}}\left(\alpha_{k}\right) S\left(\alpha_{k}, 1, T\right) d \alpha_{k}=\sum_{\ell=0}^{\infty} \frac{\Gamma\left(\frac{2 \ell+3}{2}\right) \Gamma\left(\frac{T^{2}}{2 \sigma^{2}}, \frac{2 \ell+3}{2}\right)}{\left(2 \sigma^{2}\right)^{\frac{2 \ell+1}{2}} \ell !(\ell+1) !} \int_{t=0}^{\infty} e^{-t}\left(\frac{2 \sigma^{2} \Omega_{k}}{\Omega_{k}+2 \sigma^{2}}\right) \frac{1}{\Omega_{k}} t^{\ell+1}\left(\frac{2 \sigma^{2} \Omega_{k}}{\Omega_{k}+2 \sigma^{2}}\right)^{\ell+1} d t \\
& =\sum_{\ell=0}^{\infty} \frac{\Gamma\left(\frac{2 \ell+3}{2}\right) \Gamma\left(\frac{T^{2}}{2 \sigma^{2}}, \frac{2 \ell+3}{2}\right)}{\left(2 \sigma^{2}\right)^{\frac{2 \ell+1}{2}} \ell !(\ell+1) !} \frac{1}{\Omega_{k}}\left(\frac{2 \sigma^{2} \Omega_{k}}{\Omega_{k}+2 \sigma^{2}}\right)^{\ell+2} \Gamma(\ell+2)=\sqrt{\Omega_{k}} \sum_{\ell=0}^{\infty} \frac{\Gamma\left(\ell+\frac{3}{2}\right) \Gamma\left(\frac{T^{2}}{2 \sigma^{2}}, \ell+\frac{3}{2}\right)}{\ell !}\left(\frac{1}{1+\frac{\Omega_{k}}{2 \sigma^{2}}}\right)^{\ell+2}\left(\frac{\Omega_{k}}{2 \sigma^{2}}\right)^{\ell+\frac{1}{2}} .
\end{aligned}
$$

$$
\begin{aligned}
E\left[z_{k}^{2}\right]= & \int_{0}^{\infty}\left(\alpha_{k}\right)^{2} f_{\alpha_{k}}\left(\alpha_{k}\right) S\left(\alpha_{k}, 2, T\right) d \alpha_{k} \\
= & \int_{\alpha_{k}=0}^{\infty}\left(\alpha_{k}\right)^{2} f_{\alpha_{k}}\left(\alpha_{k}\right) e^{-\frac{\alpha_{k}^{2}}{2 \sigma^{2}}} \sum_{\ell=0}^{\infty} \frac{1}{2} \frac{\left(\alpha_{k}\right)^{2 \ell}}{\ell !\left(2 \sigma^{2}\right)^{\ell}} \Gamma\left(\frac{T^{2}}{2 \sigma^{2}}, \ell+1\right) d \alpha_{k}+ \\
& \int_{\alpha_{k}=0}^{\infty}\left(\alpha_{k}\right)^{2} f_{\alpha_{k}}\left(\alpha_{k}\right) e^{-\frac{\alpha_{k}^{2}}{2 \sigma^{2}}} \sum_{\ell=0}^{\infty} \frac{1}{2} \frac{\left(\alpha_{k}\right)^{2 \ell+2}}{(\ell+2) ! \ell !\left(2 \sigma^{2}\right)^{\ell+1}} \Gamma\left(\frac{T^{2}}{2 \sigma^{2}}, \ell+2\right) \Gamma(\ell+2) d \alpha_{k} \\
= & \sum_{\ell=0}^{\infty} \frac{1}{2 \Omega_{k}} \frac{\Gamma\left(\frac{T^{2}}{2 \sigma^{2}}, \ell+1\right)}{\ell !\left(2 \sigma^{2}\right)^{\ell}}\left(\frac{2 \Omega_{k} \sigma^{2}}{\Omega_{k}+2 \sigma^{2}}\right)^{\ell+2} \Gamma(l+2)+\sum_{\ell=0}^{\infty} \frac{1}{2 \Omega_{k}} \frac{\Gamma\left(\frac{T^{2}}{2 \sigma^{2}}, \ell+2\right)}{\ell !\left(2 \sigma^{2}\right)^{\ell+1}}\left(\frac{2 \Omega_{k} \sigma^{2}}{\Omega_{k}+2 \sigma^{2}}\right)^{\ell+3} \Gamma(\ell+2) \\
= & \Omega_{k} \sum_{\ell=0}^{\infty} \frac{1}{2} \frac{\Gamma(l+2)}{\ell !}\left(\frac{\Omega_{k}}{2 \sigma^{2}}\right)^{\ell}\left(\frac{1}{1+\frac{\Omega_{k}}{2 \sigma^{2}}}\right)^{\ell+2}\left[\Gamma\left(\frac{T^{2}}{2 \sigma^{2}}, \ell+1\right)+\left(\frac{\Omega_{k}}{2 \sigma^{2}}\right)\left(\frac{1}{1+\frac{\Omega_{k}}{2 \sigma^{2}}}\right) \Gamma\left(\frac{T^{2}}{2 \sigma^{2}}, \ell+2\right)\right]
\end{aligned}
$$

$$
\begin{aligned}
E_{\theta_{e}, \hat{\alpha} \mid \alpha}\left[\frac{1}{\hat{\alpha}} \cos \theta_{e}\right] & =\int_{\alpha_{\min }}^{\infty} \int_{\pi}^{\pi} \frac{1}{\hat{\alpha}} \cos \theta_{e} f_{\theta_{e}, \hat{\alpha} \mid \alpha}\left(\hat{\alpha}, \theta_{e}\right) d \theta_{e} d \hat{\alpha}=\int_{\alpha_{\min }}^{\infty} \frac{1}{\sigma^{2}} e^{-\frac{\alpha^{2}+\hat{\alpha}^{2}}{2 \sigma^{2}}} I_{1}\left(\frac{\alpha \hat{\alpha}}{\sigma^{2}}\right) d \hat{\alpha} \\
& =e^{-\frac{\alpha^{2}}{2 \sigma^{2}}} \sum_{l=0}^{\infty} \frac{(\alpha)^{2 \ell+1}}{\ell ! \Gamma(\ell+2)\left(2 \sigma^{2}\right)^{\ell+1}} \Gamma\left(\frac{\alpha_{\min }^{2}}{2 \sigma^{2}}, \ell+1\right) \Gamma(\ell+1) .
\end{aligned}
$$

since $E\left[\left|\hat{\alpha}_{k}\right|^{2}\right]=\Omega_{k}+2 \sigma^{2}$, where $\sigma^{2}=\frac{N_{0}}{2 E_{P} N_{P}}$ as in the previous section. The received signal at the fusion center is given by (7). Dropping the time index $n$, the decision statistic at the fusion center is given by

$$
y_{R}=\sum_{k=1}^{N} x_{k} \hat{\alpha}_{k} \alpha_{k} \cos \theta_{e . k}+\eta_{R}[n]
$$

Then, the average received SNR at the FC is

$$
\gamma_{M R T}=\frac{E\left[\left|\sum_{k=1}^{N} x_{k} \hat{\alpha}_{k} \alpha_{k} \cos \theta_{e, k}\right|^{2}\right]}{\sigma_{N}^{2}}
$$

where $\sigma_{N}^{2}$ is the variance of the real part of the noise at the FC. Writing $\alpha_{k} \hat{\alpha}_{k} \cos \theta_{e, k}=u_{k}$,

$$
\begin{aligned}
\gamma_{M R T} & =\frac{1}{\sigma_{N}^{2}} E\left[\sum_{k=1}^{N} x_{k}^{2} u_{k}^{2}\right]+E\left[\sum_{j=1}^{N} \sum_{i=1, i \neq j}^{N} x_{i} x_{j} u_{i} u_{j}\right] \\
& =\frac{1}{\sigma_{N}^{2}} \sum_{k=1}^{N} E\left[x_{k}^{2}\right] E\left[u_{k}^{2}\right]+
\end{aligned}
$$

$$
\sum_{j=1}^{N} \sum_{i=1, i \neq j}^{N} E\left[x_{i} x_{j}\right] E\left[u_{i}\right] E\left[u_{j}\right]
$$

Observe that $u_{k}=\frac{r_{k}+r_{k}^{*}}{2}$, where $r_{k}=g_{k} \hat{g}_{k}^{*}$ and $g_{k}=\alpha_{k} e^{j \theta_{k}}$ and $\hat{g}_{k}=\hat{\alpha}_{k} e^{j \hat{\theta}_{k}} \cdot \hat{g}_{k}$ is the estimated channel gain given by $\hat{g}_{k}=g_{k}+w_{k}$ where $w_{k}$ is the estimation error that is circularly symmetric Gaussian with variance $2 \sigma^{2}$ and is independent of $g_{k}$. Since $E\left[r_{k}\right]=E\left[g_{k}\left(g_{k}+w_{k}\right)^{*}\right]=E\left[\left|g_{k}\right|^{2}\right]=\Omega_{k}=E\left[r_{k}^{*}\right]$, we have

$$
E\left[u_{k}\right]=\frac{2 \Omega_{k}}{2}=\Omega_{k} .
$$

Next, we compute $E\left[u_{k}^{2}\right]$.

$$
\begin{aligned}
& E\left[u_{k}^{2}\right]=E\left[\frac{\left(r_{k}+r_{k}^{*}\right)^{2}}{4}\right] \\
& =\frac{E\left[r_{k}^{2}\right]+E\left[\left(r_{k}^{*}\right)^{2}\right]+E\left[\left|r_{k}\right|^{2}\right]+E\left[\left|r_{k}^{*}\right|^{2}\right]}{4} .
\end{aligned}
$$

Now, $E\left[r_{k}^{2}\right]=E\left[g_{k}^{2}\left(g_{k}^{*}+\nu_{k}^{*}\right)^{2}\right]=E\left[\left|g_{k}\right|^{4}\right]=2 \Omega_{k}^{2}=E\left[\left(r_{k}^{*}\right)^{2}\right]$ and $E\left[\left|r_{k}\right|^{2}\right]=E\left[\left|g_{k}\right|^{2}\left(g_{k}+\nu_{k}\right)\left(g_{k}^{*}+\nu_{k}^{*}\right)\right]=E\left[\left|g_{k}\right|^{4}\right]+$ $E\left[\left|g_{k}\right|^{2}\right] E\left[\left|\nu_{k}\right|^{2}\right]=2 \Omega_{k}^{2}+\Omega_{k} 2 \sigma^{2}=E\left[\left|r_{k}^{*}\right|^{2}\right]$. Upon using 
these two in (55), we have

$$
E\left[u_{k}^{2}\right]=\frac{2 E\left[r_{k}^{2}\right]+2 E\left[\left|r_{k}\right|^{2}\right]}{4}=\Omega_{k}^{2}\left(2+\frac{\sigma^{2}}{\Omega_{k}}\right) .
$$

Using equations (54) and (56) in (53), we have $\gamma_{M R T}$ shown in (38).

\section{B. Truncated Channel Inversion}

We now analyze the performance of TCI in Section II-B2. The power allocation scheme at the sensor $i$ is given by (8). With channel estimation, the average power constraint $\bar{P}$, the fading threshold $\alpha_{\min }$, and the sensor power level $P_{0}$ are related, using (8), as

$$
\bar{P}=\int_{\alpha_{\min }}^{\infty} \frac{P_{0}}{\hat{\alpha}_{k}^{2}} \times \frac{2 \hat{\alpha}_{k} e^{-\frac{\hat{\alpha}_{k}^{2}}{\Omega_{k}+2 \sigma^{2}}}}{\Omega_{k}+2 \sigma^{2}} d \hat{\alpha}=\frac{P_{0} E_{i}\left(\frac{\alpha_{\min }{ }^{2}}{\Omega_{k}+2 \sigma^{2}}\right)}{\Omega_{k}+2 \sigma^{2}},
$$

where $E_{i}(x) \triangleq \int_{x}^{\infty} \frac{e^{-t}}{t} d t$ is the exponential integral function [26]. The value of $P_{0}$ for a given value of $\alpha_{\min }$ and $\bar{P}$ can be obtained from (57).

With the transmission strategy in (8), the average received SNR at the FC is given by (39). A closed form expression for $\bar{\gamma}_{T C I}$ can be obtained using (78) for $E\left[P\left(\hat{\alpha}_{i}\right) \alpha_{i}{ }^{2} \cos ^{2} \theta_{e, i}\right]$ and (79) for $E\left[\sqrt{P\left(\hat{\alpha}_{i}\right)} \alpha_{i} \cos \theta_{e, i}\right]$, from Appendix-D, in (39). If the channels from the $N$ sensors to the $\mathrm{FC}$ are i.i.d., and if the observations are identical, (39) simplifies to

$$
\begin{aligned}
\bar{\gamma}_{T C I}= & \frac{1}{\sigma_{N}^{2}}\left[N E\left[P(\hat{\alpha}) \alpha^{2} \cos ^{2} \theta_{e}\right]+\right. \\
& \left.N(N-1)\left(E\left[\sqrt{P(\hat{\alpha})} \alpha \cos \theta_{e}\right]\right)^{2}\right] .
\end{aligned}
$$

The performance of TCI for various fixed values of $\alpha_{\min }$ as well as with the optimum value of $\alpha_{\min }$ will be illustrated in the next section.

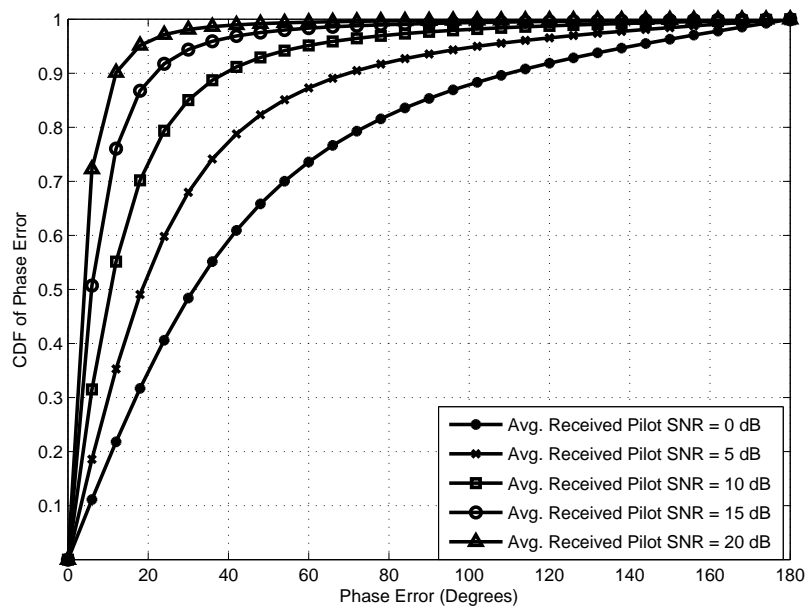

Fig. 2. Distribution of the phase error at a given sensor node on Rayleigh fading channels.

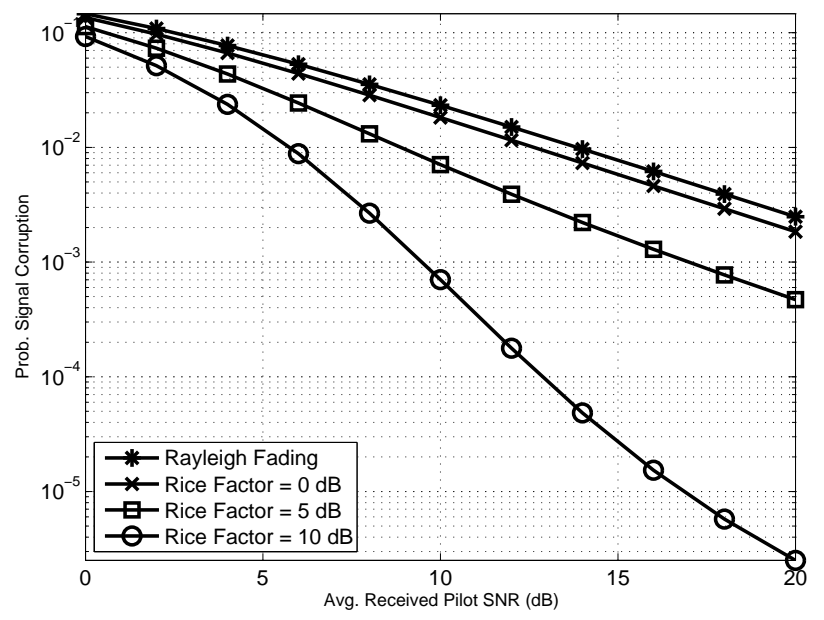

Fig. 3. Probability of signal corruption by a sensor node at the FC due to imperfect phase estimation in the downlink.

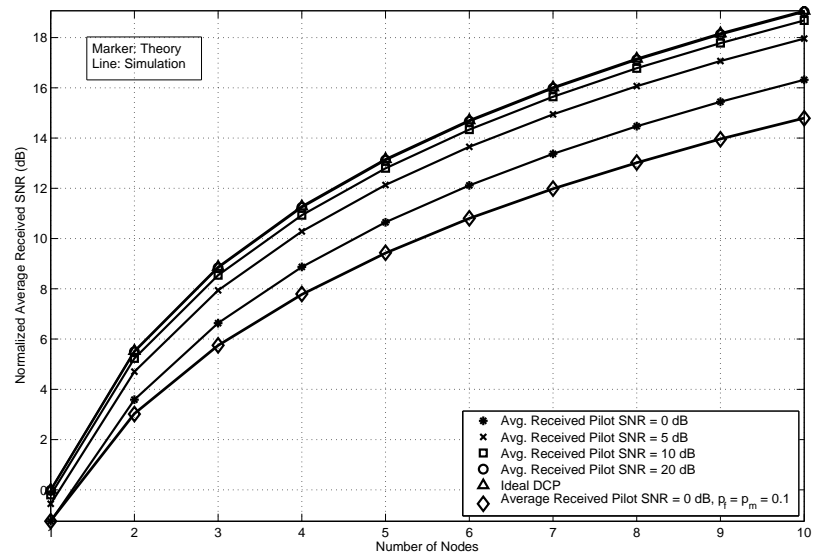

Fig. 4. Average received SNR at the FC versus the number of sensor nodes, parameterized by the downlink pilot SNR, simulation results are compared with analytical values for i.i.d. Rayleigh fading channels.

\section{RESUlTS AND DisCUSSION}

The CDF of the phase error at the sensor nodes is plotted in Fig. 2 for various values of the average received pilot SNR. As an illustration, increasing the received pilot SNR from $0 \mathrm{~dB}$ to $10 \mathrm{~dB}$ improves the probability that the phase error is less than $40^{\circ}$ by about thirty percent. The probability of signal corruption at the FC, $P_{S C}$, is calculated as derived in Section III-B and is plotted in Fig. 3. A Rayleigh fading channel as well as Rician fading channels with Rice factors of 0,5 and $10 \mathrm{~dB}$ are considered. From Fig. 3, we notice an inverse linear relationship of $P_{S C}$ with the average received pilot SNR (with unit slope at high SNR) at the sensor node for Rayleigh fading channels, as would be expected from (14). On the other hand, when a strong line-of-sight component is present, the signal corruption probability decays exponentially with the received pilot SNR.

The normalized average received SNR is plotted in Fig. 4 as a function of the number of sensor nodes participating in the data transmission phase. To simplify the simulation setup, we assume i.i.d. Rayleigh fading in the rest of this section. For various values of the received pilot SNR, the theoretical 


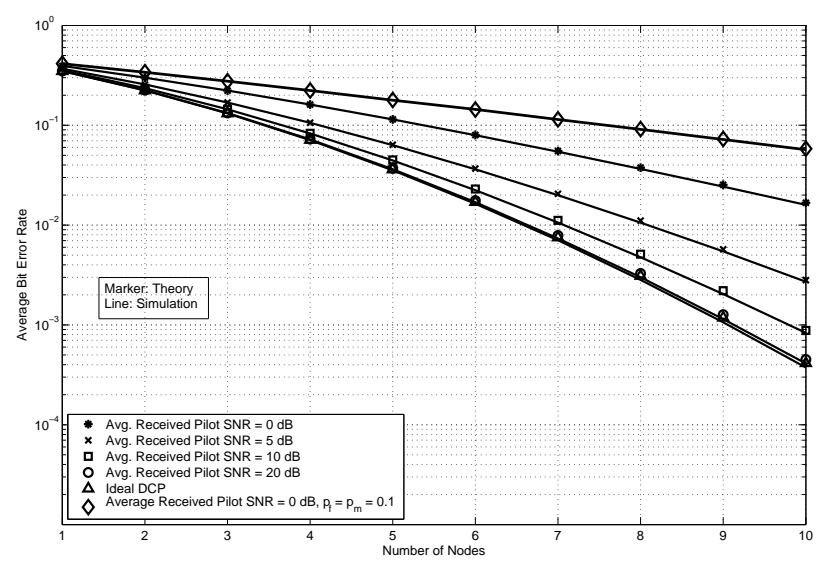

Fig. 5. Average bit error probability at the FC for Rayleigh fading channel. The average received data $\mathrm{SNR}=-10 \mathrm{~dB}$. The IGA of (21) agrees very well with the simulation results.

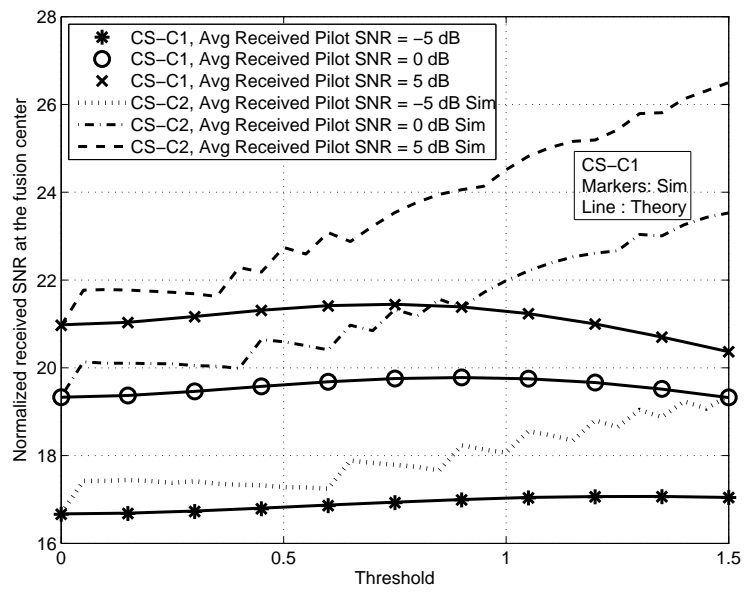

Fig. 6. Average received SNR at the FC for CS-C1 and CS-C2.

average SNR analyzed in Section III-C1 is compared against the Monte-Carlo simulations, and we see an excellent match between the two. We also see an excellent match in the theoretical and simulation-based values of average SNR when the sensor observations could be in error with probability 0.1 , with average received pilot $\mathrm{SNR}=0 \mathrm{~dB}$.

The average BER obtained from the expressions derived in Sec III-C2 is compared in Fig. 5 against the BER obtained via Monte-Carlo simulations. The average data SNR, $E_{s} / N_{0}$, is set to $-10 \mathrm{~dB}$ and the pilot SNR is varied from 0 to $20 \mathrm{~dB}$. For comparison, performance of ideal DCP (i.e., perfect phase estimation at the sensors) is also shown as a function of the number of sensor nodes. Also, the performance of DCP when the sensors' observations could be in error with probability 0.1 is plotted for the case when the pilot SNR is $0 \mathrm{~dB}$. We see the IGA is accurate over the range of pilot SNR and the size of the sensor cluster. Further, the performance gap between ideal DCP and pilot-based DCP is virtually closed with a pilot SNR of $20 \mathrm{~dB}$.

Next, the performance of the censoring-based transmission schemes in terms of average SNR received at the FC considered in section IV is plotted in Fig. 6. We compare

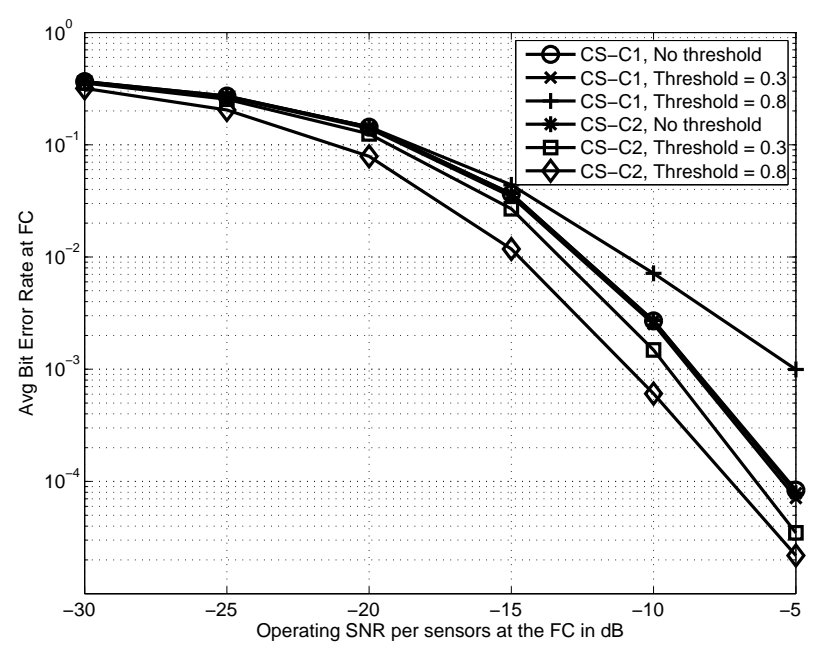

Fig. 7. Average BER at the FC using censoring at the sensors for various operating SNRs, parameterized by the threshold.

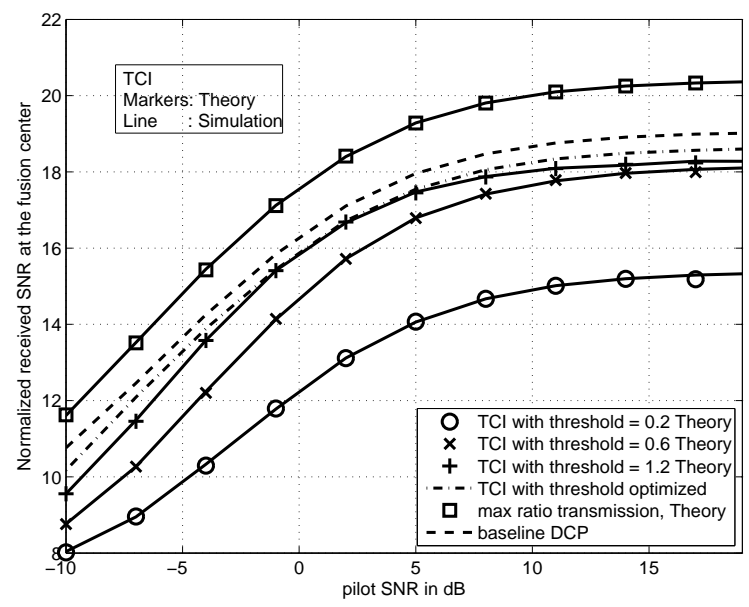

Fig. 8. Average SNR at the FC using variable power allocation schemes at the sensors. The average SNR with baseline DCP is also plotted for comparison.

the performance of $\mathrm{CS}-\mathrm{C} 1$ and $\mathrm{CS}-\mathrm{C} 2$ and also validate the analytically derived expressions for the average received SNR at the $\mathrm{FC}$ in (33). We set the number of sensors participating in $\mathrm{CS}-\mathrm{C} 1$ and the average number of transmitting sensors in $\mathrm{CS}-\mathrm{C} 2$ as ten. With the threshold set to zero, CS-C1 corresponds to the baseline DCP. We see that there is a very slight increase in the received SNR with increase in threshold till a certain threshold is reached. Thereafter, the average SNR starts to decrease since few sensors cross the threshold for very high thresholds, and hence the events where no sensor transmits starts to dominate the performance. We see that the theoretically derived values match with the simulated values, thus validating our analysis. We do not compare the CS-C2 curves with the corresponding analytically derived values as it involves evaluating the same terms as in CS-C1. The graph of average SNR due to CS-C2 is not a smooth curve because of the ceiling function in (4), due to which the number of sensors increases in a step-wise manner as the threshold is increased. The performance improves as compared to the no 


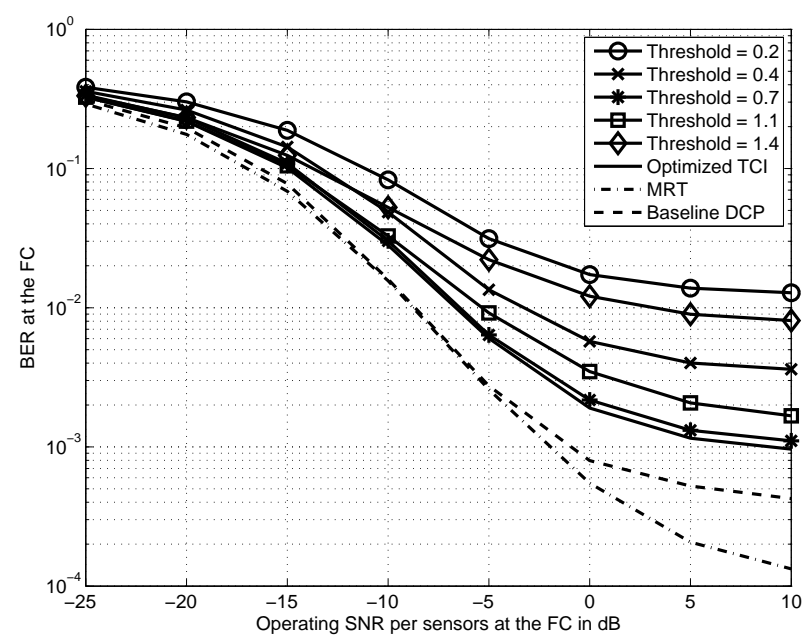

Fig. 9. Average BER at the FC using variable power allocation schemes at the sensors.

censoring scheme because the total number of cooperating sensors is being increased with increasing threshold resulting in better system performance. However, for a fixed value of $N_{\text {eff }}$, as expected, the received SNR decreases with increasing threshold. We compare the two schemes in terms of the BER at the FC in Fig. 7. We set the data SNR, $\frac{E_{s}}{N_{0}}$ to $-5 \mathrm{~dB}$. We can draw similar conclusions about CS-C1 and CS-C2 as from Fig. 7.

In Figs. 8 and 9, we compare the performance of the two variable power allocations discussed in Sec V. We consider ten sensors which participate in transmission. We compare these schemes with the baseline DCP. We see that MRT performs the best. The performance with TCI is shown for different fixed thresholds $\alpha_{\min }$. It is seen that the performance increases with threshold for a given pilot SNR and then starts to degrade. The initial performance improvement is because sensors do not expend power trying to invert bad channel states, thus saving power to transmit on the good channel states. The performance degradation at high threshold values is because the probability that no sensor transmits becomes significant and starts to dominate. When no sensor transmits, the received SNR will be zero, and the probability of error will be 0.5 . Hence, an appropriate threshold must be chosen as function of the pilot SNR to obtain optimal performance. The performance obtained by such an optimal threshold, separately optimized for maximum received SNR and for minimum BER (curves labelled TCI with threshold optimized and optimized TCI respectively) is also shown in the figures.

Finally, the performance and the relative merits of all the schemes considered in this paper are qualitatively summarized in Table I for the case of sensors with identical observations.

\section{CONCLUSION}

In this paper, we have analyzed the performance of various transmission schemes on a pilot-assisted DCP system that relies on uplink-downlink reciprocity via TDD signaling to reduce the channel feedback required to achieve co-phased transmission in a distributed manner. We derived expressions

\begin{tabular}{|l|l|l|l|}
\hline $\begin{array}{l}\text { Scheme } \\
\text { no. }\end{array}$ & $\begin{array}{l}\text { Scheme } \\
\text { Acronym }\end{array}$ & Description (Pros/Cons) & Performance \\
\hline \hline 1 & DCP & $\begin{array}{l}\text { constant power transmission; } \\
\text { estimation of channel phase }\end{array}$ & $\begin{array}{l}\text { baseline for } \\
\text { other schemes }\end{array}$ \\
\hline 2 & CS-C1 & $\begin{array}{l}\text { constant power transmission } \\
\text { for a given threshold; channel } \\
\text { estimation at sensors }\end{array}$ & $\begin{array}{l}\text { marginally } \\
\text { better than } \\
\text { DCP }\end{array}$ \\
\hline 3 & CS-C2 & $\begin{array}{l}\text { same CS-C1; number of sen- } \\
\text { sors increases with threshold }\end{array}$ & $\begin{array}{l}\text { strictly better } \\
\text { than DCP }\end{array}$ \\
\hline 4 & TCI & $\begin{array}{l}\text { channel estimation at sensors; } \\
\text { power controlled transmission }\end{array}$ & $\begin{array}{l}\text { inferior to } \\
\text { DCP }\end{array}$ \\
\hline 5 & MRT & same requirements as TCI & $\begin{array}{l}\text { superior to } \\
\text { DCP }\end{array}$ \\
\hline
\end{tabular}

TABLE I

QUALITATIVE COMPARISON OF DIFFERENT SCHEMES UNDER IDENTICAL OBSERVATIONS

for the distribution of the phase error at the sensor nodes, the probability of signal corruption at the FC due to imperfect phase estimation at the sensors and the average received SNR both for the case when the censors have a perfect detection and imperfect detection. We showed an excellent match between our proposed Gaussian approximation based average BER against the Monte-Carlo simulations. We also derived expressions for the average received SNR for a scheme based on censoring sensors, as well as for the MRT and TCI schemes, and validated them using Monte-Carlo simulations. We showed that CS with DCP does not significantly improve performance if the number of sensors transmitting is kept a constant (CS-C1), while it leads to better performance if the number of sensors is varied with the threshold, keeping the average number of transmitting sensors fixed (CS-C2). We also demonstrated that DCP with MRT improves performance both in terms of averaged received SNR and BER, whereas with DCP performs better than TCI in terms of average received SNR. Thus, DCP is a promising technique for uplink communication from low-power sensors when they have correlated data to transmit cooperatively at a fixed power level to a FC.

\section{APPENDIX A \\ DERIVATION OF $E\left[\alpha_{k} \cos \theta_{e, k}\right]$}

We write $E\left[\alpha_{k} \cos \theta_{e, k}\right]=E\left[\alpha_{k} E\left[\cos \theta_{e, k} \mid \alpha_{k}\right]\right]$. In [27], the conditional expectation $E\left[\cos \theta_{e, k} \mid \alpha_{k}\right]$ is derived as

$$
\begin{aligned}
E\left[\cos \theta_{e, k} \mid \alpha_{k}\right]= & \alpha_{k} \sqrt{\frac{\bar{\gamma}_{p} \pi}{4}} e^{-\frac{\bar{\gamma}_{p} \alpha_{k}^{2}}{2}} \times \\
& {\left[I_{0}\left(\frac{\bar{\gamma}_{p} \alpha_{k}^{2}}{2}\right)+I_{1}\left(\frac{\bar{\gamma}_{p} \alpha_{k}^{2}}{2}\right)\right] }
\end{aligned}
$$

where $I_{n}(x)$ is the modified Bessel function of the first kind of order $n$ [26]. Using (59) and setting $s=\bar{\gamma}_{p} / 2$, we simplify $E\left[\alpha_{k} \cos \theta_{e, k}\right]$ as

$$
E\left[\alpha_{k} \cos \theta_{e, k}\right]=\sqrt{\frac{s \pi}{2}} E\left[\gamma_{k} e^{-s \gamma_{k}}\left[I_{0}\left(s \gamma_{k}\right)+I_{1}\left(s \gamma_{k}\right)\right]\right] .
$$

From [26], using $I_{0}(z)=(1 / \pi) \int_{-1}^{+1} e^{-t z} / \sqrt{1-t^{2}} d t$ and $I_{1}(z)=(z / \pi) \int_{-1}^{+1} e^{-t z} \sqrt{1-t^{2}} d t$, (60) is expressed as (40). 
For the Rayleigh fading channel, using $\mathcal{L}_{\gamma_{k}}(u)=\left(1+u \Omega_{k}\right)^{-1}$, $d \mathcal{L}_{\gamma_{k}}(u) / d u=-\Omega_{k}\left(1+u \Omega_{k}\right)^{-2}$, and $d^{2} \mathcal{L}_{\gamma_{k}}(u) / d u^{2}=$ $\Omega_{k}^{2}\left(1+u \Omega_{k}\right)^{-3}$, we arrive at (41) after some simplification. Upon using the identities [28] $\int_{-1}^{+1} d t(t+a)^{-2} / \sqrt{1-t^{2}}=$ $\pi a /\left(\left(a^{2}-1\right) \sqrt{a^{2}-1}\right)$ and $\int_{-1}^{+1}(t+a)^{-3} \sqrt{1-t^{2}} d t=\pi /\left(2\left(a^{2}-\right.\right.$ 1) $\sqrt{a^{2}-1}$ ), where $a>1$, in (41), we obtain

$$
E\left[\alpha_{k} \cos \theta_{e, k}\right]=\sqrt{\frac{\pi \Omega_{k}}{4}} \sqrt{\frac{\bar{\gamma}_{p} \Omega_{k}}{1+\bar{\gamma}_{p} \Omega_{k}}} .
$$

\section{APPENDIX B \\ DERIVATION OF $E\left[\alpha_{k}^{2} \cos ^{2} \theta_{e, k}\right]$}

Using $\cos ^{2} \theta_{e, k}=\left(1+\cos 2 \theta_{e, k}\right) / 2$, $E\left[\alpha_{k}^{2} \cos ^{2} \theta_{e, k}\right]=\Omega_{k} / 2+E\left[\alpha_{k}^{2} \cos 2 \theta_{e, k}\right] / 2$, and $E\left[\alpha_{k}^{2} \cos 2 \theta_{e, k}\right]=E\left[\alpha_{k}^{2} E\left[\cos 2 \theta_{e, k} \mid \alpha_{k}^{2}\right]\right]$, we simplify $\mathcal{I}\left(\alpha_{k}\right) \triangleq E\left[\cos 2 \theta_{e, k} \mid \alpha_{k}^{2}\right]=E\left[\cos 2\left|\theta_{e, k}\right| \mid \alpha_{k}^{2}\right]$ as

$$
\mathcal{I}\left(\alpha_{k}\right)=E\left[\cos 2\left|\theta_{e, k}\right| \mid \alpha_{k}^{2}\right]=\int_{0}^{\pi} \cos 2 \theta f_{\left|\theta_{e, k}\right| \mid \alpha_{k}}\left(\theta \mid \alpha_{k}\right) d \theta \text {. }
$$

Using the following representation of $f_{\left|\theta_{e, k}\right| \mid \alpha_{k}}\left(\theta \mid \alpha_{k}\right)$ from [25]

$$
\begin{aligned}
& f_{\left|\theta_{e, k}\right| \mid \alpha_{k}}\left(\theta \mid \alpha_{k}\right)=\frac{e^{-\bar{\gamma}_{p} \alpha_{k}^{2}}}{\pi}+\frac{\bar{\gamma}_{p} \alpha_{k}^{2} \sin 2 \theta}{\pi} \times \\
& \int_{0}^{\pi-\theta} \frac{e^{-\frac{\bar{\gamma}_{p} \alpha_{k}^{2} \sin ^{2} \theta}{\sin ^{2} x}}}{\sin ^{2} x} d x, 0 \leq \theta<\pi,
\end{aligned}
$$

in (62) we obtain

$$
\begin{aligned}
\mathcal{I}\left(\alpha_{k}\right)= & \frac{e^{-\bar{\gamma}_{p} \alpha_{k}^{2}}}{\pi} \int_{0}^{\pi} \cos 2 \theta d \theta+\frac{\bar{\gamma}_{p} \alpha_{k}^{2}}{\pi} \int_{\theta=0}^{\pi} \cos 2 \theta \sin 2 \theta \\
& \times \int_{x=0}^{\pi-\theta} \frac{e^{-\frac{\bar{\gamma}_{p} \alpha_{k}^{2} \sin ^{2} \theta}{\sin ^{2} x}}}{\sin ^{2} x} d x d \theta
\end{aligned}
$$

Using $\int_{0}^{\pi} \cos 2 \theta d \theta=0$ and $\int_{\theta=0}^{\pi} \int_{x=0}^{\pi-\theta}=\int_{x=0}^{\pi} \int_{\theta=0}^{\pi-x},(64)$ reduces to (42). Using (42)

$$
\begin{aligned}
E\left[\alpha_{k}^{2} \cos ^{2} \theta_{e, k}\right] & =\frac{\Omega_{k}}{2}+\frac{1}{2} E\left[\alpha_{k}^{2} \mathcal{I}\left(\alpha_{k}\right)\right] \\
& =\frac{\Omega_{k}}{2}+\frac{1}{2} E\left[\alpha_{k}^{2}-\frac{1-e^{-\bar{\gamma}_{p} \alpha_{k}^{2}}}{\bar{\gamma}_{p}}\right] \\
& =\Omega_{k}-\frac{1-\mathcal{L}_{\gamma_{k}}\left(\bar{\gamma}_{p}\right)}{2 \bar{\gamma}_{p}} .
\end{aligned}
$$

For the Rayleigh fading channel, using $\mathcal{L}_{\gamma_{k}}(u)=\left(1+u \Omega_{k}\right)^{-1}$, (65) simplifies to

$$
E\left[\alpha_{k}^{2} \cos ^{2} \theta_{e, k}\right]=\Omega_{k}\left(1-\frac{1}{2} \times \frac{1}{1+\bar{\gamma}_{p} \Omega_{k}}\right) .
$$

\section{ApPEndix C}

DERIVATION OF $E\left[z_{k}\right]$ AND $E\left[z_{k}^{2}\right]$

Let us recall that $z_{k}=1_{\left\{\hat{\alpha}_{k}>T\right\}} \alpha_{k} \cos \theta_{e, k}$. The $k$ th moment of $z_{k}, m_{k}(n)=E\left[z_{k}^{n}\right]$, can be simplified as (43). Define $R\left(\hat{\alpha}_{k}, \alpha_{k}, n\right)=\int_{\theta=-\pi}^{\pi} \cos ^{n} \theta f_{\hat{\alpha}_{k}, \theta_{e, k} \mid \alpha_{k}}\left(\hat{\alpha}_{k}, \theta\right) d \theta$. Using (29), $R\left(\hat{\alpha}_{k}, \alpha_{k}, n\right)$ is simplified as

$$
\begin{aligned}
& R\left(\hat{\alpha}_{k}, \alpha_{k}, n\right)=\int_{\theta=-\pi}^{\pi} \frac{\cos ^{n} \theta \hat{\alpha}_{k}}{2 \pi \sigma^{2}} e^{-\frac{\left[\hat{\alpha}_{k}^{2}+\alpha_{k}^{2}-2 \alpha_{k} \hat{\alpha}_{k} \cos \theta\right]}{2 \sigma^{2}}} d \theta \\
& =\frac{e^{-\frac{\left[\hat{\alpha}_{k}^{2}+\alpha_{k}^{2}\right]}{2 \sigma^{2}}} \hat{\alpha}_{k}}{2 \pi \sigma^{2}} \int_{\theta=-\pi}^{\pi} \cos ^{n} \theta e^{\frac{\hat{\alpha}_{k} \alpha_{k} \cos \theta}{\sigma^{2}}} d \theta
\end{aligned}
$$

By expanding $\cos ^{n} \theta$ in (67) in terms of $\cos n \theta$, we have

$$
\begin{aligned}
& R\left(\hat{\alpha}_{k}, \alpha_{k}, 1\right)=\frac{\hat{\alpha}_{k}}{\sigma^{2}} e^{-\frac{\left[\hat{\alpha}_{k}^{2}+\alpha_{k}^{2}\right]}{2 \sigma^{2}}} I_{1}\left(\frac{\hat{\alpha}_{k} \alpha_{k}}{\sigma^{2}}\right), \text { and } \\
& R\left(\hat{\alpha}_{k}, \alpha_{k}, 2\right)=\frac{\hat{\alpha}_{k}}{\sigma^{2}} e^{-\frac{\left[\hat{\alpha}_{k}^{2}+\alpha_{k}^{2}\right]}{2 \sigma^{2}}} \int_{\theta=-\pi}^{\pi} \cos ^{2} \theta e^{\frac{\hat{\alpha}_{k} \alpha_{k} \cos \theta}{\sigma^{2}}} d \theta \\
& =\frac{\hat{\alpha}_{k}}{2 \sigma^{2}} e^{-\frac{\left[\hat{\alpha}_{k}^{2}+\alpha_{k}^{2}\right]}{2 \sigma^{2}}}\left[I_{0}\left(\frac{\hat{\alpha}_{k} \alpha_{k}}{\sigma^{2}}\right)+I_{2}\left(\frac{\hat{\alpha}_{k} \alpha_{k}}{\sigma^{2}}\right)\right],
\end{aligned}
$$

where, from [26],

$$
\begin{aligned}
I_{n}(x) & \triangleq \int_{-\pi}^{\pi} \cos n \theta \exp (x \cos \theta) d \theta \\
& =\left(\frac{x}{2}\right)^{n} \sum_{l=0}^{\infty} \frac{\left(\frac{x^{2}}{4}\right)^{l}}{\Gamma(l+1) \Gamma(n+l+1)} .
\end{aligned}
$$

Define

$$
S\left(\alpha_{k}, n, T\right) \triangleq \int_{\hat{\alpha}_{k}=T}^{\infty} R\left(\hat{\alpha}_{k}, \alpha_{k}, n\right) d \hat{\alpha}_{k}
$$

Using (68), (69) and (70) in (71), we obtain $S\left(\alpha_{k}, 1, T\right)$ and $S\left(\alpha_{k}, 2, T\right)$ as (44) and (45), respectively, where $\Gamma(x, n)=$ $\int_{x}^{\infty} \frac{e^{-t} t^{n-1}}{\Gamma(n)} d t$ is the incomplete Gamma function [26]. Using (44) and (45) in (43), with $f_{\alpha_{k}}(x)=2 x \exp \left(-x^{2} / \Omega_{k}\right) / \Omega_{k}$, $x \geq 0, E\left[z_{k}\right]$ is obtained in (46). In (46), the third step was obtained by substituting $t=x^{2}\left(\frac{1}{\Omega_{k}}+\frac{1}{2 \sigma^{2}}\right)$. In a similar manner, $E\left[z_{k}^{2}\right]$ is obtained in (47).

\section{APPENDIX D}

Derivation of $E\left[P\left(\hat{\alpha}_{i}\right) \alpha_{i}^{2} \cos ^{2} \theta_{e, i}\right]$ AND

$$
E\left[\sqrt{P\left(\hat{\alpha}_{i}\right)} \alpha_{i} \cos \theta_{e, i}\right]
$$

Here, we derive expressions for $E\left[P\left(\hat{\alpha}_{i}\right) \alpha_{i}^{2} \cos ^{2} \theta_{e, i}\right]$ and $E\left[\sqrt{P\left(\hat{\alpha}_{i}\right)} \alpha_{i} \cos \theta_{e, i}\right]$. Since $E\left[P(\hat{\alpha}) \alpha^{2} \cos ^{2} \theta_{e}\right]=$ $E_{\alpha}\left[\alpha^{2} E_{\theta_{e}, \hat{\alpha} \mid \alpha}\left[P(\hat{\alpha}) \cos ^{2} \theta_{e}\right]\right]$, using (8) and (29), we simplify $E_{\theta_{e}, \hat{\alpha} \mid \alpha}\left[P(\hat{\alpha}) \cos ^{2} \theta_{e}\right]$ as (72)-(74). Using (73) and (74) in (72), $E\left[P(\hat{\alpha}) \alpha^{2} \cos ^{2} \theta_{e}\right]=$ $E_{\alpha}\left[\alpha^{2} E_{\theta_{e}, \hat{\alpha} \mid \alpha}\left[P(\hat{\alpha}) \cos ^{2} \theta_{e}\right]\right]$ is obtained as

$$
\begin{aligned}
E\left[P(\hat{\alpha}) \alpha^{2} \cos ^{2} \theta_{e}\right] & =E_{\alpha}\left[\frac{P_{0}}{2 \sigma^{2}} \alpha^{2} e^{-\frac{\alpha^{2}}{2 \sigma^{2}}}\left(D_{0}+D_{1}\right)\right] \\
& =F_{0}+F_{1}
\end{aligned}
$$

where $F_{0}$ and $F_{1}$ are given by (75) and (76), respectively. 


$$
\begin{aligned}
& E_{\theta_{e}, \hat{\alpha} \mid \alpha}\left[P(\hat{\alpha}) \alpha^{2} \cos ^{2} \theta_{e}\right]=\int_{0}^{\infty} \int_{-\pi}^{\pi} 1_{\left\{\hat{\alpha}>\alpha_{\min }\right\}} P_{0} \frac{\alpha^{2}}{\hat{\alpha}^{2}} \cos ^{2} \theta_{e} f_{\hat{\alpha}, \theta_{e} \mid \alpha}\left(\hat{\alpha}, \theta_{e}\right) d \theta_{e} d \hat{\alpha} \\
& =\int_{\alpha_{\min }}^{\infty} P_{0} \frac{\alpha^{2}}{2 \pi \sigma^{2} \hat{\alpha}} e^{-\frac{\alpha^{2}+\hat{\alpha}^{2}}{2 \sigma^{2}}} \int_{-\pi}^{\pi} \cos ^{2} \theta_{e} e^{-\frac{\alpha \hat{\alpha} \cos \theta_{e}}{\sigma^{2}}} d \theta_{e} d \hat{\alpha}=\int_{\alpha_{\min }}^{\infty} P_{0} \frac{\alpha^{2}}{2 \pi \sigma^{2} \hat{\alpha}} e^{-\frac{\alpha^{2}+\hat{\alpha}^{2}}{2 \sigma^{2}}}\left[I_{0}\left(\frac{\alpha \hat{\alpha}}{\sigma^{2}}\right)+I_{2}\left(\frac{\alpha \hat{\alpha}}{\sigma^{2}}\right)\right] d \hat{\alpha} \\
& =\frac{P_{0}}{2 \sigma^{2}} \alpha^{2} e^{-\frac{\alpha^{2}}{2 \sigma^{2}}}\left[\int_{\alpha_{\min }}^{\infty} \frac{1}{\hat{\alpha}} e^{-\frac{\hat{\alpha}}{2 \sigma^{2}}} I_{0}\left(\frac{\alpha \hat{\alpha}}{\sigma^{2}}\right) d \hat{\alpha}+\int_{\alpha_{\min }}^{\infty} \frac{1}{\hat{\alpha}} e^{-\frac{\hat{\alpha}^{2}}{2 \sigma^{2}}} I_{2}\left(\frac{\alpha \hat{\alpha}}{\sigma^{2}}\right) d \hat{\alpha}\right]=\frac{P_{0}}{2 \sigma^{2}} \alpha^{2} e^{-\frac{\alpha^{2}}{2 \sigma^{2}}}\left(D_{0}+D_{1}\right), \\
& \text { where } \quad D_{0}=\int_{\alpha_{\min }}^{\infty} \frac{1}{\hat{\alpha}} e^{-\frac{\hat{\alpha}}{2 \sigma^{2}}} I_{0}\left(\frac{\alpha \hat{\alpha}}{\sigma^{2}}\right) d \hat{\alpha}=\sum_{l=0}^{\infty} \frac{(\alpha)^{2 \ell}}{\left(2 \sigma^{2}\right)^{2 \ell} \ell ! \Gamma(\ell+1)} \int_{\alpha_{\min }}^{\infty} \hat{\alpha}^{2 \ell-1} e^{-\frac{\hat{\alpha}^{2}}{2 \sigma^{2}}} d \hat{\alpha}=\sum_{l=0}^{\infty} \frac{\left(\frac{\alpha^{2}}{2 \sigma^{2}}\right)^{\ell}}{2 \ell ! \Gamma(\ell+1)} \Gamma\left(\frac{\alpha_{\min }^{2}}{2 \sigma^{2}}, \ell\right) \Gamma(\ell) \\
& \text { and } \quad D_{1}=\int_{\alpha_{\min }}^{\infty} \frac{1}{\hat{\alpha}} e^{-\frac{\hat{\alpha}^{2}}{2 \sigma^{2}}} I_{2}\left(\frac{\alpha \hat{\alpha}}{\sigma^{2}}\right) d \hat{\alpha}=\sum_{l=0}^{\infty} \frac{\left(\frac{\alpha^{2}}{2 \sigma^{2}}\right)^{\ell+1}}{2 \ell ! \Gamma(\ell+3)} \Gamma\left(\frac{\alpha_{\min }^{2}}{2 \sigma^{2}}, \ell+1\right) \Gamma(\ell+1) \text {. }
\end{aligned}
$$

$$
\begin{aligned}
F_{0} & =\int_{0}^{\infty} \frac{P_{0}}{2 \sigma^{2}} \alpha^{2} e^{-\frac{\alpha^{2}}{2 \sigma^{2}}} \sum_{l=0}^{\infty} \frac{\left(\frac{\alpha^{2}}{2 \sigma^{2}}\right)^{\ell}}{2 \ell ! \Gamma(\ell+1)} \Gamma\left(\frac{\alpha_{\min }^{2}}{2 \sigma^{2}}, \ell\right) \Gamma(\ell) f_{\alpha}(\alpha) d \alpha \\
& =\frac{P_{0}}{2} \sum_{l=0}^{\infty} \frac{\Gamma\left(\frac{\alpha_{\min }^{2}}{2 \sigma^{2}}, \ell\right) \Gamma(\ell)}{\ell ! \Gamma(\ell+1)\left(2 \sigma^{2}\right)^{\ell+1}} \int_{0}^{\infty} \frac{2 \alpha}{\Omega}\left(\alpha^{2}\right)^{\ell+1} e^{-\frac{\alpha^{2}}{2 \sigma^{2}}} e^{-\frac{\alpha^{2}}{\Omega}} d \alpha=\frac{P_{0}}{2 \Omega} \sum_{l=0}^{\infty} \frac{\Gamma\left(\frac{\alpha_{\min }^{2}}{2 \sigma^{2}}, \ell\right) \Gamma(\ell)}{\ell ! \Gamma(\ell+1)\left(2 \sigma^{2}\right)^{\ell+1}}\left(\frac{2 \Omega \sigma^{2}}{2 \sigma^{2}+\Omega}\right)^{\ell+2} \int_{0}^{\infty} x^{\ell+1} e^{-x} d x \\
& =\frac{P_{0} / 2}{\Omega / 2 \sigma^{2}} \sum_{l=0}^{\infty} \frac{\Gamma\left(\frac{\alpha_{\min }^{2}}{2 \sigma^{2}}, \ell\right) \Gamma(\ell) \Gamma(\ell+2)}{\ell ! \Gamma(\ell+1)}\left(\frac{\frac{\Omega}{2 \sigma^{2}}}{1+\frac{\Omega}{2 \sigma^{2}}}\right)^{\ell+2} .
\end{aligned}
$$

$$
\begin{aligned}
F_{1} & =\int_{0}^{\infty} \frac{P_{0}}{2 \sigma^{2}} \alpha^{2} e^{-\frac{\alpha^{2}}{2 \sigma^{2}}} \sum_{l=0}^{\infty} \frac{\left(\frac{\alpha^{2}}{2 \sigma^{2}}\right)^{\ell+1}}{2 \ell ! \Gamma(\ell+3)} \Gamma\left(\frac{\alpha_{\min }^{2}}{2 \sigma^{2}}, \ell+1\right) \Gamma(\ell+1) f_{\alpha}(\alpha) d \alpha \\
& =\frac{P_{0}}{2} \sum_{l=0}^{\infty} \frac{\Gamma\left(\frac{\alpha_{\min }^{2}}{2 \sigma^{2}}, \ell+1\right) \Gamma(\ell+1)}{\ell ! \Gamma(\ell+3)\left(2 \sigma^{2}\right)^{\ell+2}} \int_{0}^{\infty}\left(\alpha^{2}\right)^{\ell+2} e^{-\frac{\alpha^{2}}{2 \sigma^{2}}} f_{\alpha}(\alpha) d \alpha=\frac{P_{0}}{2 \Omega} \sum_{l=0}^{\infty} \frac{\Gamma\left(\frac{\alpha_{\min }^{2}}{2 \sigma^{2}}, \ell+1\right) \Gamma(\ell+1)}{\ell ! \Gamma(\ell+3)\left(2 \sigma^{2}\right)^{\ell+2}}\left(\frac{2 \Omega \sigma^{2}}{2 \sigma^{2}+\Omega}\right)^{\ell+3} \int_{0}^{\infty} x^{\ell+2} e^{-x} d x \\
& =\frac{P_{0} / 2}{\Omega / 2 \sigma^{2}} \sum_{l=0}^{\infty} \frac{\Gamma\left(\frac{\alpha_{\min }^{2}}{2 \sigma^{2}}, \ell+1\right) \Gamma(\ell+1)}{\ell !}\left(\frac{\frac{\Omega}{2 \sigma^{2}}}{1+\frac{\Omega}{2 \sigma^{2}}}\right)^{\ell+3} .
\end{aligned}
$$

$$
\begin{aligned}
E\left[\sqrt{P_{0}} \frac{\alpha}{\hat{\alpha}} \cos \theta_{e}\right] & =E_{\alpha}\left[\sqrt{P_{0}} \alpha e^{-\frac{\alpha^{2}}{2 \sigma^{2}}} \sum_{l=0}^{\infty} \frac{(\alpha)^{2 \ell+1} \Gamma(\ell+1)}{\ell !\left(2 \sigma^{2}\right)^{\ell+1}} \Gamma\left(\frac{\alpha_{\min }^{2}}{2 \sigma^{2}}, \ell+1\right)\right] \\
& =\sqrt{P_{0}} \sum_{l=0}^{\infty} \frac{\Gamma\left(\frac{\alpha_{\min }^{2}}{2 \sigma^{2}}, \ell+1\right) \Gamma(\ell+1)}{\ell ! \Gamma(\ell+2)\left(2 \sigma^{2}\right)^{\ell+1}} \int_{0}^{\infty}(\alpha)^{2 \ell+2} e^{-\frac{\alpha^{2}}{2 \sigma^{2}}} f_{\alpha}(\alpha) d \alpha \\
& =\frac{\sqrt{P_{0}}}{\Omega / 2 \sigma^{2}} \sum_{l=0}^{\infty} \Gamma\left(\frac{\alpha_{\min }^{2}}{2 \sigma^{2}}, \ell+1\right)\left(\frac{\frac{\Omega}{2 \sigma^{2}}}{1+\frac{\Omega}{2 \sigma^{2}}}\right)^{\ell+2} .
\end{aligned}
$$

$$
\begin{aligned}
& E\left[\sqrt{P(\hat{\alpha})} \alpha \cos \theta_{e}\right]=\frac{\sqrt{P_{0}}}{\Omega / 2 \sigma^{2}} \sum_{l=0}^{\infty} \Gamma\left(\frac{\alpha_{\min }^{2}}{2 \sigma^{2}}, \ell+1\right)\left(\frac{\frac{\Omega}{2 \sigma^{2}}}{1+\frac{\Omega}{2 \sigma^{2}}}\right)^{\ell+2} \\
& E\left[P(\alpha) \alpha^{2} \cos ^{2} \theta_{e}\right]=\frac{P_{0} / 2}{\Omega / 2 \sigma^{2}}\left[\sum_{l=0}^{\infty} \frac{\Gamma\left(\frac{\alpha_{\min }^{2}}{2 \sigma^{2}}, \ell\right) \Gamma(\ell) \Gamma(\ell+2)}{\ell ! \Gamma(\ell+1)}\left(\frac{\frac{\Omega}{2 \sigma^{2}}}{1+\frac{\Omega}{2 \sigma^{2}}}\right)^{\ell+2}+\sum_{l=0}^{\infty} \frac{\Gamma\left(\frac{\alpha_{\min }^{2}}{2 \sigma^{2}}, \ell+1\right) \Gamma(\ell+1)}{\ell !}\left(\frac{\frac{\Omega}{2 \sigma^{2}}}{1+\frac{\Omega}{2 \sigma^{2}}}\right)^{\ell+3}\right] .
\end{aligned}
$$

We now evaluate $E\left[\sqrt{P_{0}} \frac{\alpha}{\tilde{\alpha}} \cos \theta_{e}\right]$. The derivation follows along the lines presented above, so only the key steps are shown here. Since $E\left[\sqrt{P_{0}} \frac{\alpha}{\alpha} \cos \theta_{e}\right]=$ $E_{\alpha}\left[E_{\theta_{e}, \hat{\alpha} \mid \alpha}\left[\sqrt{P_{0}} \frac{\alpha}{\hat{\alpha}} \cos \theta_{e}\right]\right]$, using $E_{\theta_{e}, \hat{\alpha} \mid \alpha}\left[(1 / \hat{\alpha}) \cos \theta_{e}\right]$ calculated in (48), we find $E\left[\sqrt{P_{0}} \frac{\alpha}{\tilde{\alpha}} \cos \theta_{e}\right]$ in (77). In summary, we have $E\left[\sqrt{P(\hat{\alpha})} \cdot \alpha \cos \theta_{e}\right]$ and $E\left[P(\alpha) \alpha^{2} \cos ^{2} \theta_{e}\right]$ as shown in (78) and (79), respectively.

\section{REFERENCES}

[1] I. F. Akyildiz, W. Su, Y. Sankarasubramaniam, and E. Cayirci, "Wireless sensor networks: a survey," Elsevier Computer Networks, vol. 38, no. 4, 15th March, pp. 393-422.

[2] J-F. Chamberland and V. V. Veeravalli, "Wireless sensors in distributed detection applications," IEEE Signal Processing Magazine, May 2007, pp. 16-25.

[3] Y.-S. Tu and G. Pottie, "Coherent cooperative transmission from multiple adjacent antennas to a distant stationary antenna through AWGN chan- 
nels," in Proc. 55th IEEE Vehicular Technology Conference (Spring), vol. 1, pp. 130-134, 2002.

[4] R. Mudumbai, G. Barriac, and U. Madhow, "On the feasibility of distributed beamforming in wireless networks," IEEE Trans. on Wireless Commun., vol. 6, no. 5, pp. 1754-1763, May 2007.

[5] H. Ochiai, P. Mitran, H. V. Poor, and V. Tarokh, "Collaborative beamforming for distributed wireless ad hoc sensor networks," IEEE Trans. on Signal Processing, vol. 53, no. 11, pp. 4110-4124, Nov. 2005.

[6] M. Ahmed and S. Vorobyov, "Collaborative beamforming for wireless sensor networks with Gaussian distributed sensor nodes," IEEE Trans. on Wireless Commun., vol. 8, no. 2, pp. 638-643, Feb. 2009.

[7] L. Dong, A. P. Petropulu, and H. V. Poor, "A cross-layer approach to collaborative beamforming for wireless ad hoc networks," IEEE Trans. Signal Process., vol. 56, no. 7, pp. 2981-2993, Jul. 2008

[8] L. Dong, A. P. Petropulu, and H. V. Poor, "Weighted cross-layer cooperative beamforming for wireless networks," IEEE Trans. on Signal Processing, vol. 57, no. 8, pp. 3240-3252, Aug. 2009.

[9] Y. Jing and H. Jafarkhani, "Network beamforming using relays with perfect channel information," Network Beamforming Using Relays With Perfect Channel Information, vol. 55, no. 6, pp. 2499-2517, Jun. 2009.

[10] V. Havary-Nassab, S. Shahbazpanahi, A. Grami and Z-Q. Luo, "Distributed beamforming for relay networks based on second-order statistics of the channel state information," IEEE Trans on Signal Processing, vol. 56, no. 9, pp. 4306-4316, Sep. 2008.

[11] E. Koyuncu, Y. Jing and H. Jafarkhani, "Distributed beamforming in wireless relay networks with quantized feedback," IEEE Journal on Selected Areas in Communications, vol. 26, no. 8, pp. 1429-1439, Oct. 2008.

[12] D. R. Brown III, G. Prince, and J. McNeill, "A method for carrier frequency and phase synchronization of two autonomous cooperative transmitters," in Proc. of the IEEE 6th Workshop on Signal Processing Advances in Wireless Communications (SPAWC 2005), New York, NY, pp. 260-264, Jun. 5-8, 2005.

[13] I. Ozil and D. R. Brown III, "Time-slotted round-trip carrier synchronization," in Proc. of the 41st Asilomar Conference on Signals, Systems, and Computers, Pacific Grove, CA, pp. 1781-1785, Nov. 4-7, 2007.

[14] R. Mudumbai, J. Hespanha, U. Madhow, G. Barriac, "Distributed transmit beamforming using feedback control," IEEE Transactions on Information Theory, vol.56, no.1, pp.411-426, Jan. 2010

[15] R. Mudumbai, D. R. Brown III, U. Madhow, and H.V. Poor, "Distributed transmit beamforming: challenges and recent progress" IEEE Communications Magazine, vol. 47, no. 2, pp. 102-110, Feb. 2009.

[16] P. Fertl, A. Hottinen, G. Matz, "Perturbation-based distributed beamforming for wireless relay networks," Proc. of the IEEE Global Communications Conference (Globecom), 2008. pp. 1-5, 2008.

[17] C. Lin, V. V. Veeravalli and S.P. Meyn, "Distributed beamforming with feedback: convergence analysis." Submitted to the IEEE Trans. on Info. Theory, July 2008. Available online: http://arxiv.org/abs/0806.3023.

[18] J. A. Bucklew ,W. A. Sethares, "Convergence of a class of decentralized beamforming algorithms," IEEE Transactions on Signal Processing, vol. 56, no. 6, Jun. 2008.

[19] S. Berger and A. Wittneben, "Carrier phase synchronization of multiple distributed nodes in a wireless network," in Proc. Signal Processing Advances in Wireless Communications, 2007, pp. 1-5, 2007.

[20] J. Holtzman, "A simple, accurate method to calculate spread-spectrum multiple-access error probabilities," IEEE Trans. Communications, vol. 40, no. 3, pp. 461-464, Mar. 1992.

[21] C. Rago, P. Willett and Y. Bar-Shallom, "Censoring sensors: a lowcommunication-rate scheme for distributed detection," IEEE Trans. on Aerospace and Electronic Systems, vol. 32, no. 2, pp. 554-568, Apr. 1996.

[22] T. K. Y. Lo, "Maximum ratio transmission," IEEE Trans. Communication, vol. 47, pp. 1458-1461, Oct. 1999.

[23] A. Goldsmith and P. P. Varaiya, "Capacity of fading channels with channel side information," IEEE Trans. on Information Theory, vol. 43, no. 6, pp. 1986-1992, Nov. 1997.

[24] J. G. Proakis, Digital Communications, Fourth Edition, McGraw-Hill, 2001.

[25] F. S. Weinstein, "Simplified relationships for the probability distribution of the phase of a sine wave in narrow-band normal noise," IEEE Trans. Info. Theory, vol. 20, no. 5, pp. 658-661, Sept. 1974.

[26] M. Abramowitz and I. A. Stegun, Handbook of Mathematical Functions, Applied Mathematics Series 55. New York: National Bureau of Standard, 1964.

[27] K. J. Quirk, "The effects of estimation errors on direct sequence spread spectrum receiver performance," Ph.D Thesis, University of California, San Diego, 2000.
[28] I. S. Gradshteyn and I. M. Ryzhik, Table of Integrals, Series and Products, 5th ed. San Diego, CA; Academic, 1994.

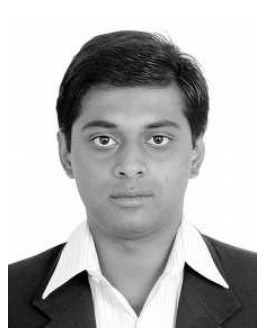

Krishna Chaythanya K. V. received his Bachelors degree in Electronics and Communication Engineering from the PES Institute of Technology (PESIT, Bangalore) in 2008, and is currently pursuing his M.Sc. (Engg.) at the Indian Institute of Science (IISc, Bangalore). His research interests are in the areas of application of signal processing and information theory for wireless communications.

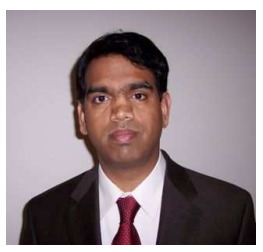

Ramesh Annavajjala is a Principal Member Research Staff at the Mitsubishi Electric Research Labs (MERL) in Cambridge, MA, USA. He has also held industry positions at ArrayComm LLC (San Jose, CA), Synopsys Inc (Bangalore, India) and CMC R \& D Center (Hyderabad, India). He received Bachelors in Electronics and Communication Engineering from the National Institute of Technology (NIT), Warangal, India, in May 1998, Masters in Telecommunications from the Indian Institute of Science (IISc), Bangalore, India, in Jan. 2001, and PhD in Electrical Engineering from the University of California at San Diego (UCSD), La Jolla, CA, in June 2006.

Dr. Annavajjala was a recipient of the Purkayastha/TimeLine Ventures graduate fellowship (2002-2003), recipient of the best paper award from the IEEE WPMC 2009 conference, and was a guest editor for the special issue "Wireless Cooperative Networks" of the EURASIP Journal on Advanced Signal Processing. His current research interests lie in the areas of statistical and array signal processing, interference management and coordinated transmission for future wireless networks, distributed sensing, communication and control for smart grid systems, delay-constrained multimedia multicast with applications to vehicular networks, and architecture and design of multi-core networks-on-chips for low-power data centers.

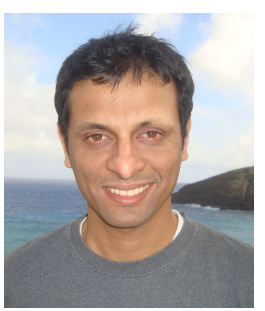

Chandra R. Murthy (S'03-M'06) received the B.Tech. degree in Electrical Engineering from the Indian Institute of Technology, Madras in 1998, the M.S. and Ph.D. degrees in Electrical and Computer Engineering from Purdue University and the University of California, San Diego, in 2000 and 2006, respectively.

From 2000 to 2002, he worked as an engineer for Qualcomm Inc., where he worked on WCDMA baseband transceiver design and $802.11 \mathrm{~b}$ baseband receivers. From Aug. 2006 to Aug. 2007, he worked as a staff engineer at Beceem Communications Inc. on advanced receiver architectures for the 802.16e Mobile WiMAX standard. In Sept. 2007, he joined as an assistant professor at the Department of Electrical Communication Engineering at the Indian Institute of Science, where he is currently working. His research interests are primarily in the areas of digital signal processing, information theory, estimation theory, and their applications in the optimization of MIMO, OFDM and CDMA wireless communication systems. Currently, he is working on Cognitive Radio, Energy Harvesting Wireless Sensors and MIMO systems with finite-rate channel-state feedback. 